\title{
Characterization of Optical Fiber Strength Under Applied Tensile Stress and Bending Stress
}

Federal Manufacturing \& Technologies

P.E. Klingsporn

KCP-613-6655

Published August 2011

Final Report

Approved for public release; distribution is unlimited.

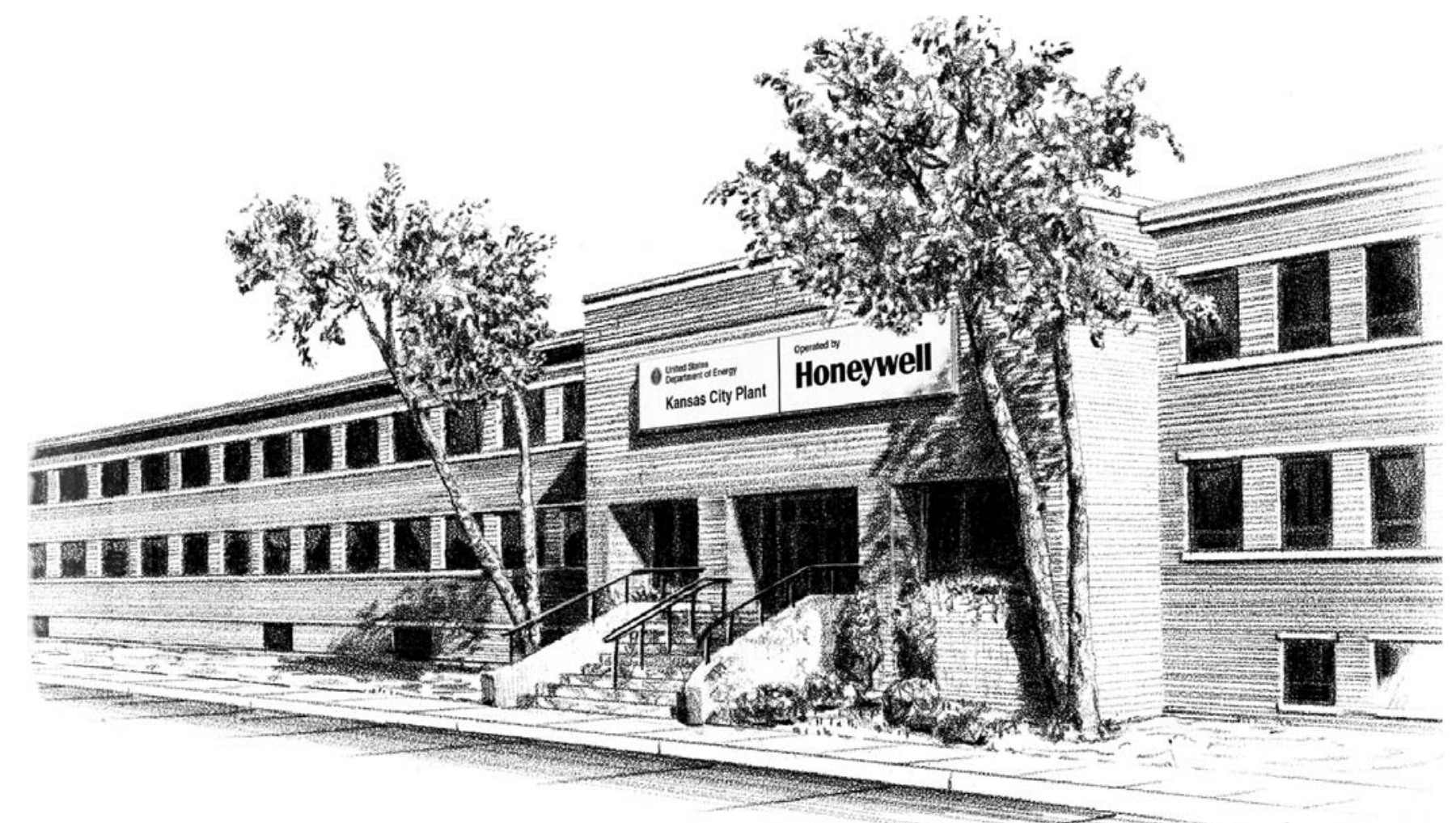

Prepared under prime contract DE-NA0000622 for the

United States Department of Energy 


\section{DISCLAIMER}

This report was prepared as an account of work sponsored by an agency of the United States Government. Neither the United States Government nor any agency thereof, nor any of their employees, makes any warranty, express or implied, or assumes any legal liability or responsibility for the accuracy, completeness, or usefulness of any information, apparatus, product, or process disclosed, or represents that its use would not infringe privately owned rights. Reference herein to any specific commercial product, process or service by trade names, trademark, manufacturer, or otherwise, does not necessarily constitute or imply its endorsement, recommendation or favoring by the United States Government or any agency thereof. The views and opinions of authors expressed herein do not necessarily state or reflect those of the United States Government or any agency thereof.

All data prepared, analyzed and presented has been developed in a specific context of work and was prepared for internal evaluation and use pursuant to that work authorized under the reference contract. Reference herein to any specific commercial product, process or service by trade name, trademark, manufacturer, or otherwise, does not necessarily constitute or imply its endorsement, recommendation or favoring by the United States Government, any agency thereof or Honeywell Federal Manufacturing \& Technologies, LLC.

Printed in the United States of America.

This report has been reproduced from the best available copy.

Available to DOE and DOE contractors from the Office of Scientific and Technical Information, P.O. Box 62, Oak Ridge, Tennessee 37831; prices available from (865) 576-8401, FTS 626-8401.

Available to the public from the National Technical Information Service, U.S. Department of Commerce, 5285 Port Royal, Rd., Springfield, Virginia 22161, (703) 487-4650.

A prime contractor with the United States Department of Energy under Contract Number DE-NA0000622
Honeywell Federal Manufacturing \& Technologies

P.O. Box 419159

Kansas City, Missouri, 64141-6159 


\title{
Honeywell
}

KCP-613-6655

Distribution Category UC-42

Approved for public release; distribution is unlimited.

\section{CHARACTERIZATION OF OPTICAL FIBER STRENGTH} UNDER APPLIED TENSILE STRESS AND BENDING STRESS

\author{
P.E. Klingsporn
}

Published August 2011

Final Report

P.E. Klingsporn, Project Leader

Project Team:

G.F. Couser 



\section{Contents}

Section Page

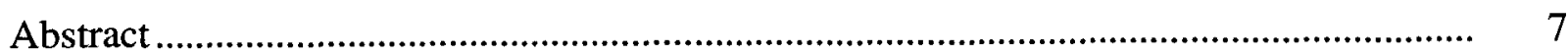

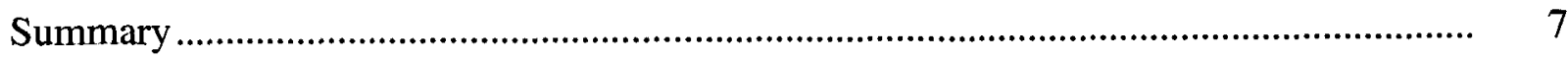

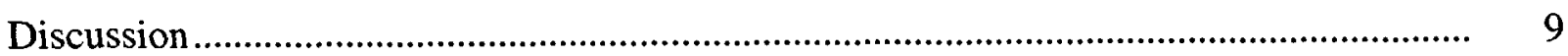

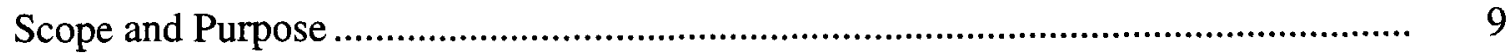

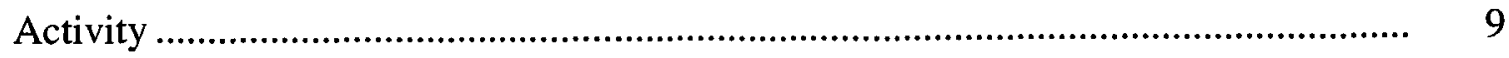

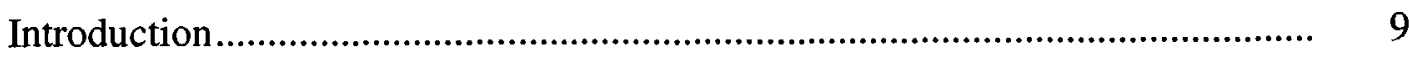

Fiber Strength-Analytic Relationships.................................................. 11

Tensile Testing ................................................................................. 14

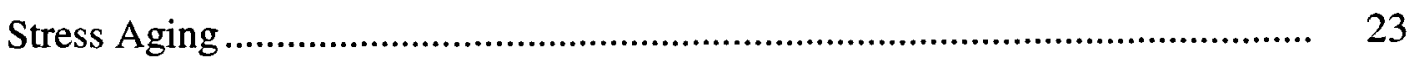

Tensile Testing of Laminated Fiber .......................................................... 29

Elastic Modulus ........................................................................... 32

Time-to-Failure Under Applied Bending Stress ......................................... 33

Tabulation of Results ....................................................................... 37

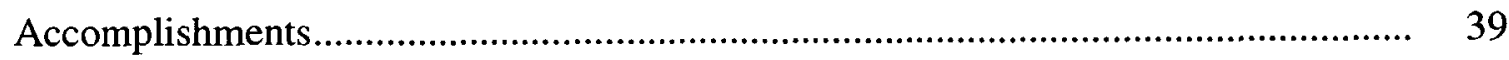

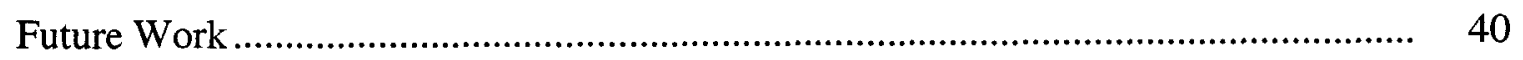

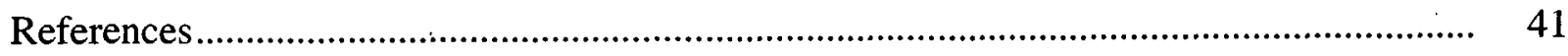

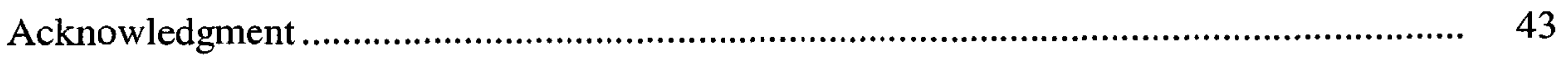




\section{Illustrations}

Figure Page

1 Schematic Representation of Optical Fiber Manufacturing................................... 10

2 Capstans Used to Secure Ends of Optical Fiber for Tensile Testing the Free Fiber Length in Between...................................................................... 15

3 Weibull Cumulative Failure Probability Versus Logarithm of Fracture Stress for $100-\mu m$ Core Fiber .................................................................................... 16

4 Weibull Cumulative Failure Probability Versus Logarithm of Fracture Stress at Two Gauge Lengths for 100- $\mu m$ Core Fiber.

5 Weibull Cumulative Failure Probability Versus Logarithm of Fracture Stress for 275- $\mu m$ Core Fiber With Polyimide Buffer

6 Weibull Cumulative Failure Probability Versus Logarithm of Fracture Stress for 200- $\mu m$ Core Fiber With Acrylate Buffer

7 Weibull Cumulative Failure Probability Versus Logarithm of Fracture Stress for $3 \mathrm{M}$ Fiber (365- $\mu \mathrm{m}$ core) and SpecTran Fiber (350- $\mu \mathrm{m}$ core) Having Different Buffer Coatings

8 Weibull Cumulative Failure Probability Versus Logarithm of Fracture Stress at Two Gauge Lengths for 3M Fiber.

9 Weibull Cumulative Failure Probability Versus Logarithm of Fracture Stress at Two Gauge Lengths for SpecTran Fiber.

10 Weibull Cumulative Failure Probability Versus Logarithm of Fracture Stress for 3M Fiber After Aging at 493.8 Kpsi Bend Stress for 170 Hours at Relative Humidity of 23 Percent, $70-72^{\circ} \mathrm{F}$

11 Effects of Proof-Test Aging on Weibull Cumulative Failure Probability, Versus Logarithm of Fracture Stress, for 3M Fiber

12 Effect of Tensile Strain Rate on Weibull Cumulative Failure Probability, Versus Logarithm of Fracture Stress, on 200- $\mu m$ Core Fiber. 
13 Schematic Representation of a Laminated Optical Fiber Segment ........................... 31

14 Method for Inducing Bending Stress in Fiber and Monitoring Time to Failure........... 34

15 Plot of Logarithm of Mean Time-to-Failure Versus Logarithm of Applied Bending Stress for 3M Fiber, 365- $\mu m$ Core Diameter............................................ 35

\section{Tables}

Number

Page

1 Compilation of Results Obtained From Tensile Testing ..................................... 38

2 Extrapolated Minimum Fiber Bend Radius for 20-Year Lifetime........................... 39 


\begin{abstract}
Various types of tensile testing and bend radius tests were conducted on silica core/silica cladding optical fiber of different diameters with different protective buffer coatings, fabricated by different fiber manufacturers. The tensile tests were conducted to determine not only the average fiber strengths at failure, but also the distribution in fracture strengths, as well as the influence of buffer coating on fracture strength. The times-to-failure of fiber subjected to constant applied bending stresses of various magnitudes were measured to provide a database from which failure times of 20 years or more, and the corresponding minimum bend radius, could be extrapolated in a statistically meaningful way. The overall study was done to provide an understanding of optical fiber strength in tensile loading and in applied bending stress as related to applications of optical fiber in various potential configurations for weapons and enhanced surveillance campaigns
\end{abstract}

\title{
Summary
}

A number of investigations were conducted on various diameters of glass optical fiber obtained from different manufacturers to determine the strength of the fiber in tensile loading and its timeto-failure under constant applied bending stresses of various magnitudes. Fiber with 100- $\mu \mathrm{m}$ core diameter and 125- $\mu \mathrm{m}$ cladding diameter manufactured by Polymicro Technologies and by SpecTran were tensile-tested to failure. The average measured stress at failure for SpecTran fiber with a Tefzel buffer coating was 12.5 percent higher than that of Polymicro fiber with an acrylate buffer. The tests were done with a standard strain rate of 4 percent of length per minute, with a 1.0- $m$ free fiber test length. Both fibers were also tested at 2.5- $m$ free lengths, with no observable difference in average failure strength. Fiber with a $275-\mu m$ core diameter manufactured by Polymicro, containing a polyimide (Kapton-like) buffer coating, was tensiletested to failure; and it was found that the average tensile stress at failure was slightly higher than that of the acrylate-coated fiber, but the variability or distribution in stress was much greater, as indicated by the Weibull slope of 11-21, compared to higher slope of 45 for the acrylate and Tefzel (fluorinated polymer)-coated fiber.

Comparative tensile failure tests were done on larger core diameter fibers obtained from SpecTran and from $3 \mathrm{M}$ for the project. The $3 \mathrm{M}$ fiber contained a protective Tefzel (fluorinated polymer) buffer coating, while the SpecTran fiber contained a carbon coating as a moisture barrier applied directly on the silica cladding by chemical vapor deposition, with a final polyimide coating. The average failure strength of the $3 \mathrm{M}$ fiber was 82 percent higher than that of the SpecTran fiber. Moreover, the variation in strength of the $3 \mathrm{M}$ fiber was much less than that of the SpecTran fiber, as evidenced by Weibull slopes of 26 and 
139 for SpecTran and 3M fiber, respectively. Evidently, carbon is not effective as a moisture barrier, or its application introduces micro-surface defects that otherwise degrade the fiber strength. Testing of both $3 \mathrm{M}$ and SpecTran fiber at free fiber lengths of $0.5 \mathrm{~m}$ and $2.5 \mathrm{~m}$ showed slightly lower strengths at the 2.5-m gauge length for $3 \mathrm{M}$ and significantly lower for SpecTran at $2.5 \mathrm{~m}$.

Effects of stress aging were conducted with the $3 \mathrm{M}$ fiber. In one testing phase, a group of fibers was subjected to a constant applied bending stress at 70 percent of the average tensile test strength. The fibers were maintained at 23 percent relative humidity at the constant bending stress for a period of 170 hours at $70-72^{\circ} \mathrm{F}$. At the end of this period of stress conditioning, each fiber was tensile-tested to failure. Not only did the average strength decrease by 12 percent (from $705 \mathrm{Kpsi}$ to $625 \mathrm{Kpsi}$ ), but the Weibull slope after aging was only 22.5, compared to 122.6 for the non-aged fiber, which indicated a much greater variation in strength after aging.

A series of other aging tests was done with the $3 \mathrm{M}$ fiber under tensile loading. These showed that the fiber strength is weakened when the fiber is subjected to high tensile loads in both static and dynamic pre-conditioning states.

A series of tests was conducted in which the $3 \mathrm{M}$ fiber was subjected to constant applied bending stress until breakage occurred. Six different bending stress levels were used, and ten fiber samples were used at each stress level. Each fiber was wrapped over a 1-m length at the given rod diameter used to create the desired bending stress, and all fibers were maintained immersed in water until fiber failure occurred to create a worst-case environment for penetration of moisture through the Tefzel buffer. The mean time-to-failure ranged from approximately 30 minutes at the highest bending stress to 1 year at the lowest bending stress. A best-fit linear relationship of the logarithm of time-to-failure versus logarithm of applied bending stress was obtained with a correlation factor of 99.92 percent. An extrapolation based on the linear relation shows that a 20 -year fiber lifetime is expected at the stress associated with a minimum bend radius of $5.968 \mathrm{~mm}$. Self-consistency of the data was verified by obtaining a best-fit linear relation to the data from 30 minutes to 30 days, omitting the data at 1 year. An extrapolation with the latter data based on five bending stress levels showed that a fiber survival time of 20 years is expected at a minimum bend radius of $6.007 \mathrm{~mm}$, which is within 0.65 percent of the value obtained from the extrapolation based on data from all six stress levels. 


\section{Discussion}

\section{Scope and Purpose}

Combinations of tensile testing and bending stress-induced tests were conducted on different diameter optical fibers, with different protective coatings, manufactured by different fiber vendors. Various types of stress-aging effects were studied. The work was done to gain an understanding of mechanical strength variability of optical fiber and the effects of environment as they relate to potential applications in optical firing systems designs and enhanced surveillance campaign activity.

\section{Activity}

\section{Introduction}

The following is a brief description of how optical fibers having diameters as small as only 125 $\mu m$ ( $0.0049 \mathrm{inch}$ ), with lengths of hundreds of meters, are produced. A silica preform, approximately 1 to 2 inches in diameter and 3 to 4 feet in length, is positioned vertically at the top of a "draw tower," and its lower portion is moved in a controlled manner into the heated region of an electric furnace, as indicated schematically in Figure 1. Under a downward force, the heated portion of the preform undergoes an elongation with a simultaneous reduction in diameter. A few feet below the furnace, the fiber passes through a non-contact laser-based system for very accurate measurements of the fiber diameter. Below that, desired protective coatings are applied as the fiber continues its descent, and then the coated region passes through another laser-based diameter measurement system. Finally, the coated fiber passes onto a takeup reel. During the drawing process, if the bare fiber diameter is measured above the upper limit of the specification, then the downward force on the fiber is increased so that, other variables being constant, the fiber diameter is drawn faster and its diameter decreased to within the desired range. Conversely, the opposite kind of drawing control is exercised when an in-process fiber diameter measurement shows the diameter to be too small. For multimode step index fiber, the annular portion of the silica preform contains a dopant that reduces its refractive index slightly below that of the pure silica; and this region becomes the cladding of the fiber, after drawing. 


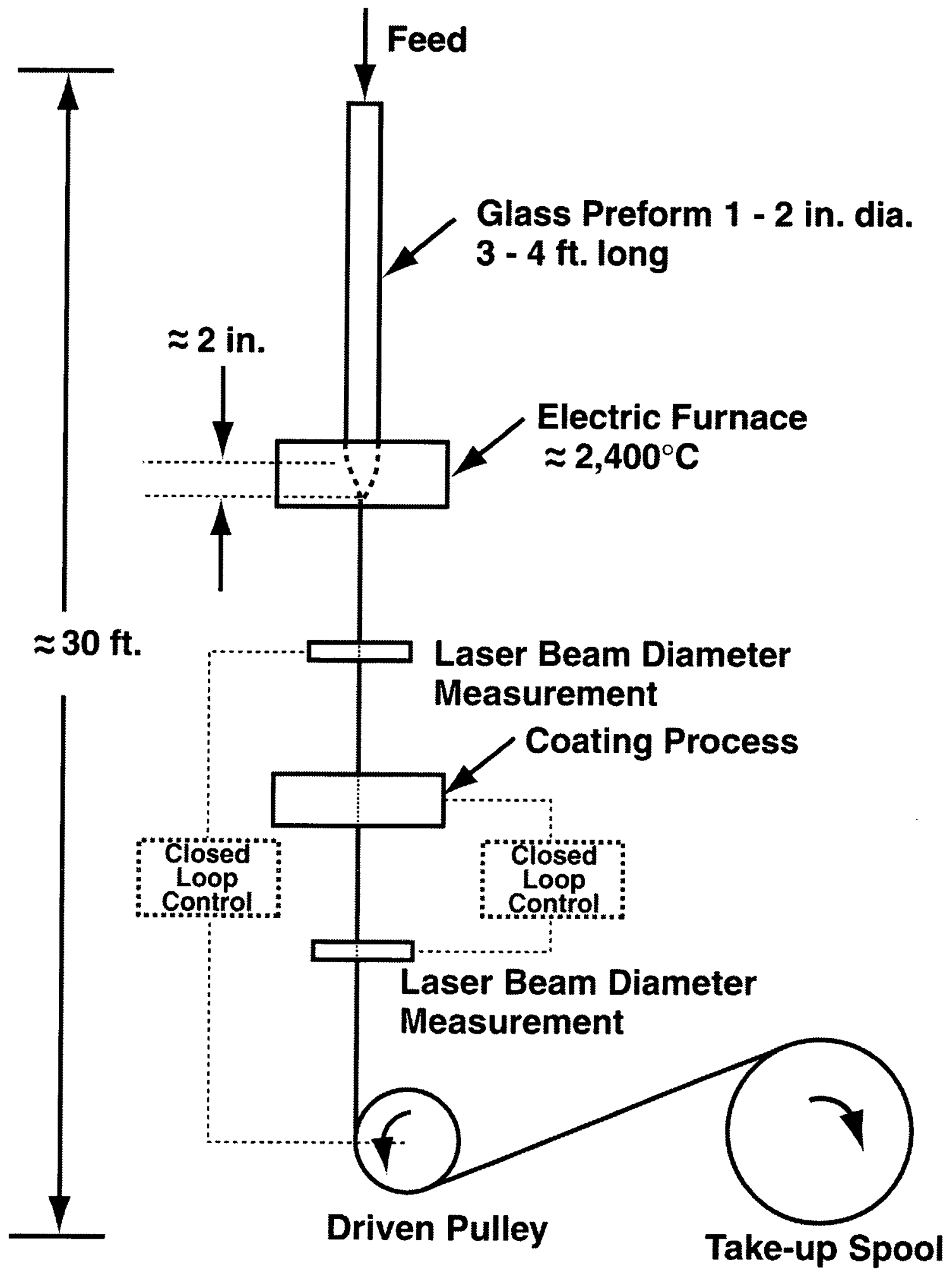

Figure 1. Schematic Representation of Optical Fiber Manufacturing 


\section{Fiber Strength - Analytic Relationships}

Although the manufacturing process for the preform is very tightly controlled and the glass is very pure, inherent surface defects are present on the drawn fiber. The glass is brittle and, under tensile stress or bending stress, surface defects in the form of minute cracks will grow, leading to fracture of the fiber. By the nature of their existence, the minute surface defects are randomly distributed in size and, therefore, the externally applied tensile or bending stress at which a given fiber length will fracture varies in magnitude. Stated another way, a given length of fiber is no stronger than its weakest link; that is, the fiber can be no stronger than that allowed by the largest surface defect which can grow under stress and lead to fracture. Also, it is well known that the presence of moisture at a minute surface defect site is particularly harmful because of stress corrosion and associated weakening of atomic bonds in the silica structure. ${ }^{1}$

A two-parameter Weibull distribution has been widely used to analyze the statistical behavior of optical fiber fracture strength. The Weibull distribution function representing the cumulative probability of failure is given $b^{2,3}$

$$
F\left(\sigma_{f}\right)=1-e^{-\left(\frac{\sigma_{f}}{\sigma_{o}}\right)^{m}}
$$

where $\sigma_{f}$ is the stress at failure in the fiber cross section, $m$ is the so-called "Weibull slope," and $\sigma_{o}$ is the characteristic strength. Equation 1 can be put into the following form.

$$
e^{\left(\frac{\sigma_{f}}{\sigma_{\mathrm{o}}}\right)^{m}}=\frac{1}{1-F\left(\sigma_{f}\right)}
$$

Taking the natural logarithms of both sides of Equation 2 yields

$$
\left(\frac{\sigma_{f}}{\sigma_{o}}\right)^{m}=\ln \frac{1}{1-F\left(\sigma_{f}\right)} \text {, }
$$

and taking natural logarithm of both sides of Equation 3 gives

$$
\ln \ln \left(\frac{1}{1-F\left(\sigma_{f}\right)}\right)=m \ln \sigma_{f}-m \ln \sigma_{o} .
$$


Let $N$ samples of fiber, each of equal length, be stressed in tensile loading to the point of failure, and let $k$ be the rank or order in which failure occurs, from the lowest to the highest stress in the fiber cross section. For brittle failure such as is the case with glass optical fiber, the cumulative failure probability expression others have found most consistent for $N$ samples is given by ${ }^{4}$

$$
F=\frac{k-0.5}{N}
$$

If $\ln \ln (1 /(1-F))$ is plotted versus $\ln \sigma_{f}$, the result should be a straight line of slope $m$ and intercept $-m \ln \sigma_{o}$, according to Equation 4. If $N$ is sufficiently large, a set of data should yield a linear relationship according to Equation 4, characterized by a single value of slope $m$ provided that the fractures all result from intrinsic surface flaws. If other surface flaws such as those from handling damage are present, then a plot of fiber failure data may show two distinct regions, one characterized by a slope $m_{s}$, characteristic of surface handling damage, and the other region of slope $m$, representative of intrinsic surface flaws.

If a segment of optical fiber is exposed to a constant applied stress, whether tensile or in bending, surface flaws such as minute cracks will grow in size over time. Eventually, the fiber will fracture. If the stress is high, fracture will occur relatively soon. For lower stress levels, failure will occur later in time. Depending upon the level of low stress, failure may not occur for years. A question of concern in many fiber applications is the lifetime before failure occurs in a fiber subjected to a given tensile or bending stress. An analytical framework that is useful in providing answers to fiber lifetime under stress is often referred to as the "power law" of crack propagation. Any given minute crack on the surface will undergo a velocity of propagation over time, under stress, given by the relation ${ }^{5,6}$

$$
\frac{d a}{d t}=A K_{I}^{n},
$$

where $K_{I}$ is a stress intensity factor and $A$ and $n$ are parameters of the so-called "power law." The stress intensity factor, $K_{l}$, is the stress level at the tip of the crack of depth $a$, and it is related to the stress $\sigma$ by the relation

$$
K_{I}=Y \sigma \sqrt{a},
$$

where $Y$ is a factor depending on the characteristic geometry of the flaw under stress. Combining Equations 6 and 7 gives

$$
d a=A Y^{n} \sigma^{n} a^{n / 2} d t
$$


Let $t_{f}$ be the time interval under constant applied stress $\sigma$ at which the fiber breaks. If $a_{o}$ is the initial crack size at time $t=o$, then the crack will have grown to $a$ after the time interval $t_{f}$, and hence Equation 8 can be integrated to give

$$
\int_{a_{o}}^{a} a^{-n / 2} d a=\int_{o}^{t_{f}} A Y^{n} \sigma^{n} d t
$$

or

$$
\left(\frac{2}{2-n}\right)\left[a^{1-n / 2}-a_{o}^{1-n / 2}\right]=A Y^{n} \sigma^{n} t_{f} .
$$

Before stress is applied, let $\sigma_{o}$ represent the initial or "inert" fiber strength corresponding to the minute crack size $a_{o}$. Then, from Equation 7, $a_{o}$ can be related to $\sigma_{o}$ through the relation $a_{o}=K_{l}^{2} / Y^{2} \sigma_{o}^{2}$. Similarly, when the crack has grown to dimension $a$, the relation $a=K_{I}^{2} / Y^{2} \sigma^{2}$ is obtained. When the expressions for $a$ and $a_{o}$ are substituted into Equation 9 , the term inside the square bracket is found to be proportional to $\sigma^{n-2}-\sigma_{o}^{n-2}$. Empirically, $n$ for silica generally has a value in the range 20 to 30 . The values of applied stress $\sigma$ that are of interest are usually much less than the inert stress $\sigma_{o}$ that a fiber can withstand. Therefore, $\sigma^{n-2}<<\sigma_{o}^{n-2}$ and $\sigma^{n-2}$ can be neglected in relation to $\sigma_{o}^{n-2}$. (In fact, as an example, if a typical value $n=22$ is taken and an assumed constant applied stress $\sigma$ equal to 70 percent of the inert stress $\sigma_{o}$ is assumed, then $\sigma^{n-2}$ is less than 0.08 percent of the value $\sigma_{o}^{n-2}$.) Hence, after considerable manipulation, Equation 9 can be solved for the lifetime $t_{f}$ as a function of the constant applied stress and expressed in the form

$$
t_{f}=B \sigma_{o}^{n-2} \sigma^{-n}
$$

where $B=\frac{2 K_{I}^{2-n}}{A Y^{2}(n-2)}$.

Taking the natural logarithm of both sides of Equation 10 gives

$$
\ln t_{f}=C-n \ln \sigma
$$

where $C$ is a constant, $C=\ln \left(B \sigma_{o}^{n-2}\right)$. 
Let a group of fibers be subjected to different constant applied stresses, and let the times to failure, $t_{f}$, be measured for each stress. According to Equation 12, a plot $\ln t_{f}$ versus $\ln \sigma$ should yield a straight line with negative slope of magnitude $n$. Of course, at any given applied stress level, a number of fibers must be tested because variations in time-to-failure will exist as a result of the variation in the initial intrinsic defect sizes present on the fiber surfaces.

\section{Tensile Testing}

\section{Fiber with 100- $\mu m$ Core Diameter}

Optical fiber with 100- $\mu m$ step-index core manufactured by two different companies was tested for comparison of strength. The first, designated by FVA 100/125/250 $\mu \mathrm{m}$, was manufactured by Polymicro Technologies, Phoenix, Arizona. Its silica core and silica cladding diameters were $100 \mu \mathrm{m}$ and $125 \mu \mathrm{m}$, and the protective buffer coating on the cladding was acrylate, with an outer diameter of $250 \mu \mathrm{m}$. The other fiber in the study was manufactured by SpecTran, Avon, Connecticut; and it had silica core and silica cladding of the same diameters as the Polymicro fiber. Its designation was $100 / 125 / 140 / 250 \mu \mathrm{m}$, wherein $140 \mu \mathrm{m}$ refers to the outer diameter of a polymer hard coat on the cladding and $250 \mu \mathrm{m}$ is the outer diameter of a Tefzel (fluorinated polymer) buffer. For testing, 20 samples of each fiber were cut, each to a length of 88 inches. This allowed 24 inches on each end to be wrapped on a capstan for securing in the Instron, with a free length of fiber in between of 1 meter. A representation of the capstans is given in Figure 2. A free fiber length of one meter is a commonly used gauge length in fiber optic testing procedures $^{7}$. The strain rate during the testing is another part of standardized testing, and the rate of 4 percent of the free length per minute is the standard. Accordingly, each fiber in turn was mounted in the Instron with a free length of 1 meter between the capstans, and tensile stress was applied at the rate of $1.6 \mathrm{in} . / \mathrm{min}$ until the fiber fractured. The Instron is a precision machine that applies the load along the fiber axis and provides a measure of fiber elongation versus load. The applied load at failure was recorded, and the stress in the silica fiber cross section (125- $\mu \mathrm{m}$ diameter) was calculated. Next, for each group of 20 fibers, the cumulative failure $F$ was calculated from Equation 5, ranking the fibers in order of failure with $k=1$ for the fiber that failed at the lowest stress and so on, with $k=20$ for the fiber that failed at the highest applied stress. A plot of the Weibull cumulative probability of failure, versus the natural logarithm of the applied stress at failure, from Equation 4, is given in Figure 3 for both types of optical fiber. The mean stress at failure is higher $(681 \mathrm{Kpsi}$ ) for the SpecTran fiber than for the Polymicro fiber (605 Kpsi). In calculating the failure stress, the outer silica cladding diameter was used, ignoring the buffer diameter. This is entirely valid because the elastic modulus of the buffer materials is orders of magnitude less than that for the silica. 


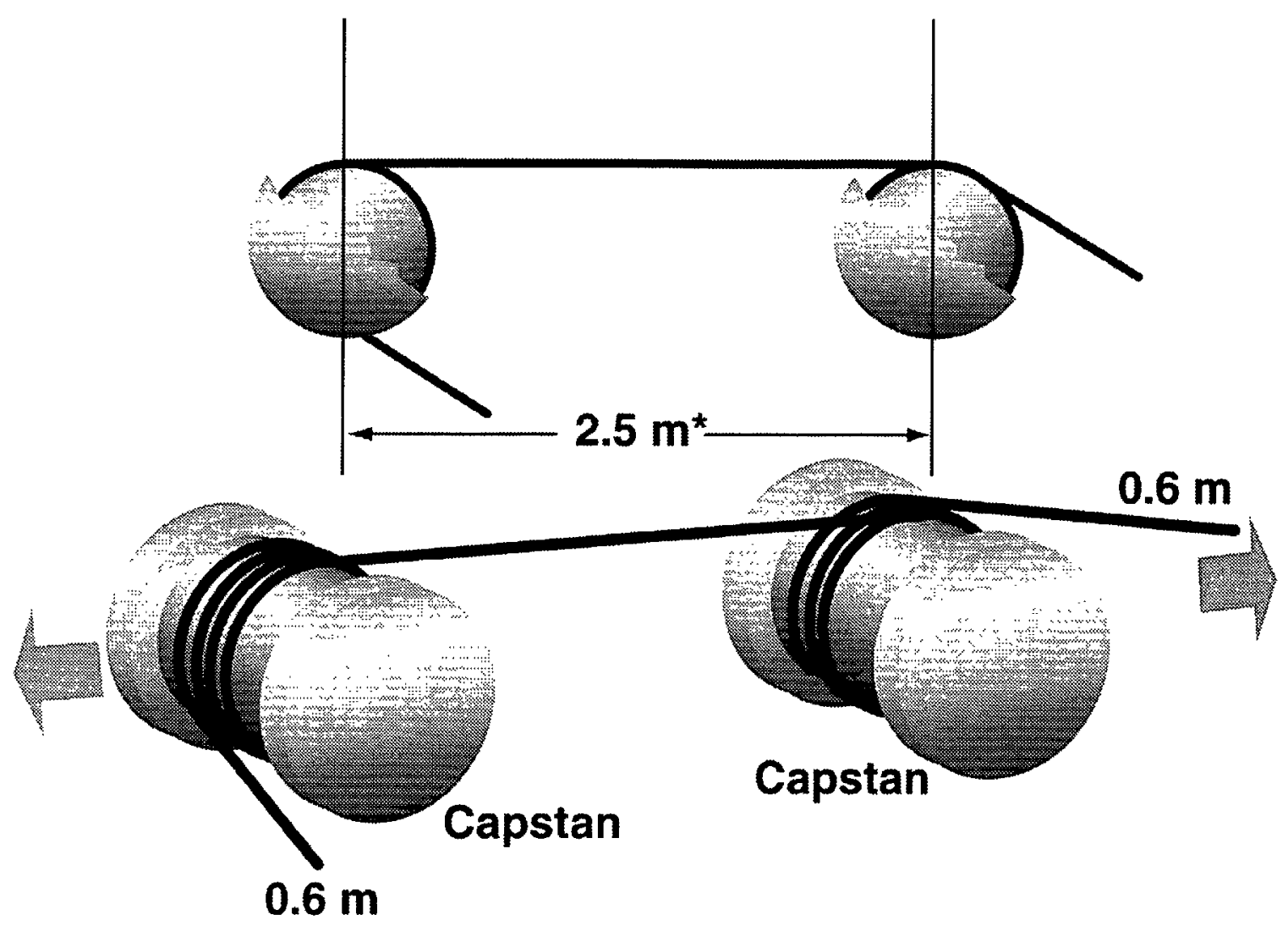

*Shorter free-fiber lengths as required

Figure 2. Capstans Used to Secure Ends of Optical Fiber for Tensile Testing the Free Fiber Length in Between

Although 1 meter is a standard free-fiber test length, 0.5 meter is often used, as well as greater lengths. ${ }^{7}$ For longer lengths, a greater sampling of the initial surface flaw defects may be activated under applied stress, depending on the flaw distribution along the fiber length.

However, because proof-testing by the vendor at the level of $100,000 \mathrm{psi}$ tends to eliminate large defects from the distribution, testing of lengths somewhat longer than 1 meter may not be expected to yield significantly different results. In the present case, weapons applications are not expected to involve fiber lengths exceeding a couple meters. Therefore, a length of 2.5 meters was chosen as a representative longer test length. Accordingly, fibers were cut to lengths of 148 inches, allowing 24 inches to be wound on the Instron capstans, at each end, giving $2.5 \mathrm{~m}$ as a free test length in between. Each fiber was strained at the standard rate of $4 \mathrm{in} . / \mathrm{min}$ ( 4 percent of test length per minute) until tensile failure occurred, and the load at failure was recorded. ${ }^{7}$ In the case of the Polymicro fiber, ten samples of $2.5 \mathrm{~m}$ in length were tested, and four samples of the SpecTran fiber were tested. Plots of the Weibull cumulative failure probability versus logarithm of tensile stress at failure are given in Figure 4 for the 2.5 meter test lengths, superimposed on the data of Figure 3, respectively, for the Polymicro and SpecTran fibers. From the data, no apparent difference exists between the test lengths of $2.5 \mathrm{~m}$ compared to test lengths of $1 \mathrm{~m}$, considering the mean tensile stress at failure. 


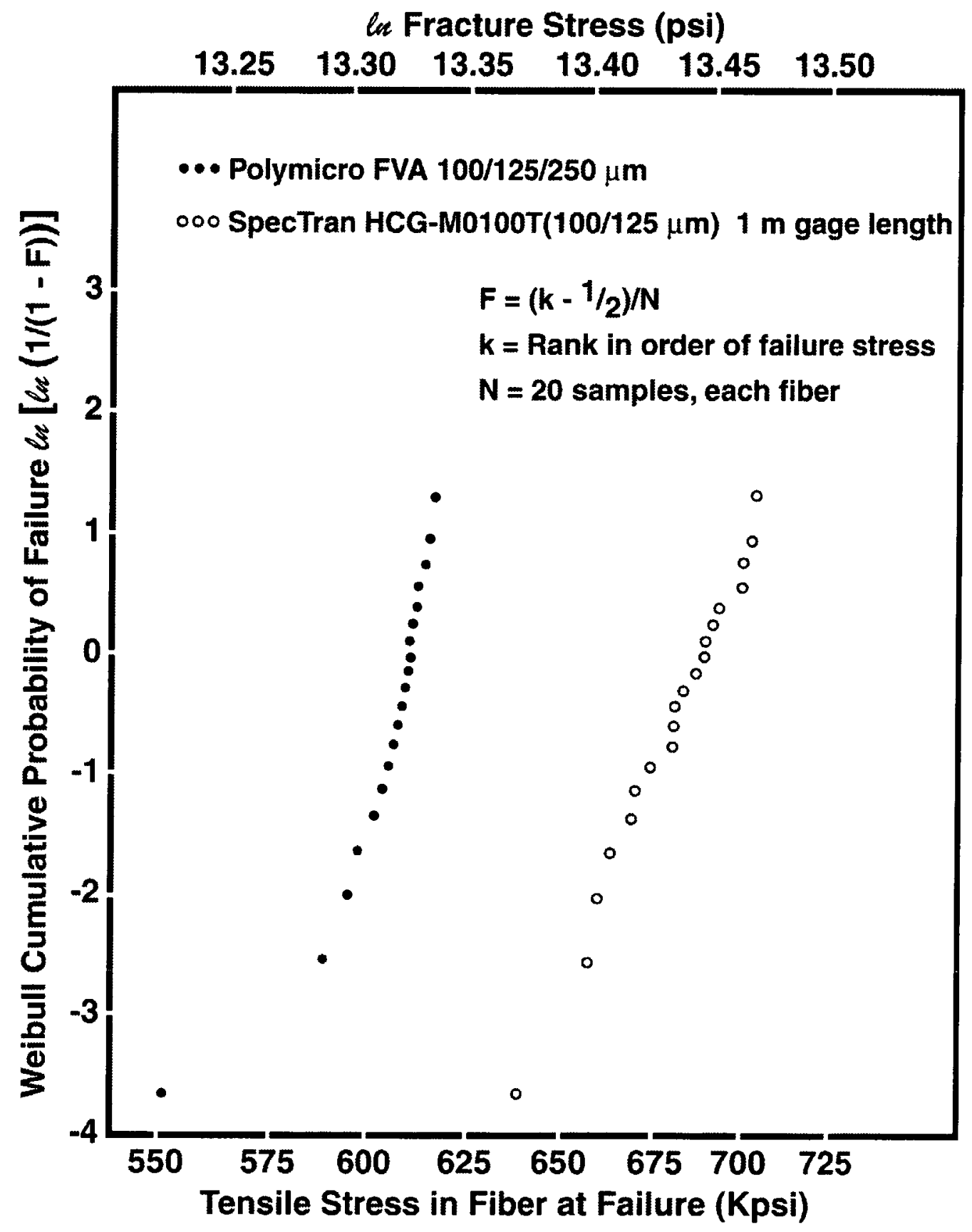

Figure 3. Weibull Cumulative Failure Probability Versus Logarithm of Fracture Stress for 100- $\mu \mathrm{m}$ Core Fiber 


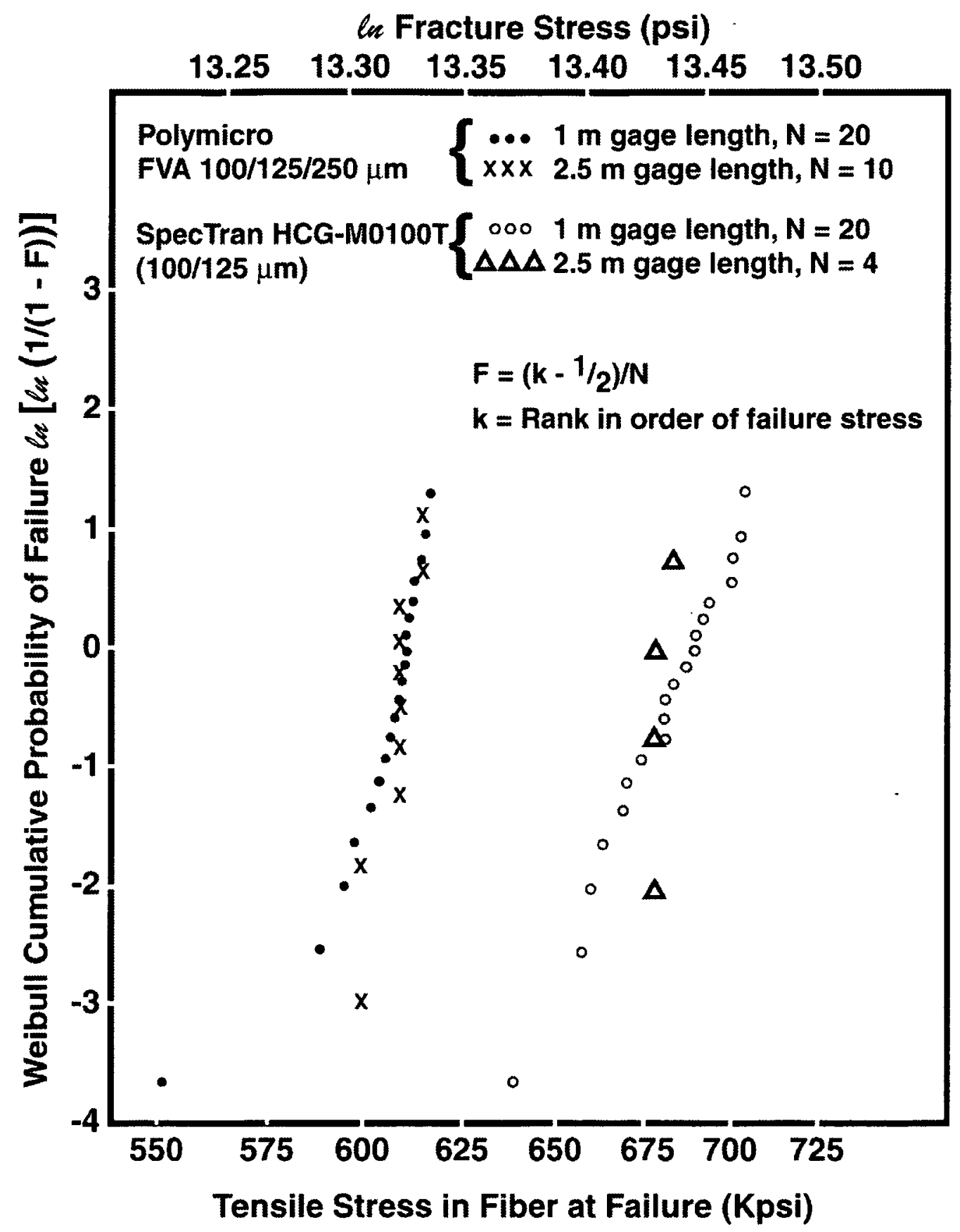

Figure 4. Weibull Cumulative Failure Probability Versus Logarithm of Fracture Stress at Two Gauge Lengths for 100- $\mu \mathrm{m}$ Core Fiber 


\section{Fiber with $275-\mu m$ Core Diameter}

Note that the buffer diameter is approximately twice the silica cladding diameter for both the SpecTran fiber and the Polymicro fiber. This is true because acrylate-type buffer materials cannot be applied uniformly for wall thicknesses less than approximately $65 \mu \mathrm{m}$. Another buffer material that can be applied with a thinner wall thickness during the fiber manufacturing process is a Kapton-like material known as polyimide. For some applications, it is necessary to have as large a silica core diameter as possible, but as small a buffer diameter as possible for passage of the composite through confined spaces. In particular, for such applications, a polyimide-coated fiber can be desirable. Accordingly, fiber with a core diameter of $275 \mu \mathrm{m}$, cladding diameter of $302 \mu \mathrm{m}$, and a polyimide buffer diameter of $340 \mu \mathrm{m}$ was procured from Polymicro Technologies for evaluation on the project. A total of eighteen fiber samples was tensile-tested to failure using a 1-meter free-fiber test length. A plot of the Weibull failure probability versus the natural logarithm of the tensile stress in the fiber at failure is given in Figure 5. The average tensile stress at failure (653 Kpsi for the fiber removed from a loose outer jacket) is slightly greater than the tensile stress at failure in the 100- $\mu m$ core fiber (see Figure 3 ) with acrylate buffer, but the Weibull slope for the polyimide-coated fiber is 20.7 , which is less than half the slope for the fiber with acrylate buffer. Apparently, the polyimide buffer is not as effective as acrylate in preventing moisture from getting to the silica surface, and/or the nature of the polyimide coating process is not as conducive to high strength as acrylate. Although the average strength of the polyimide-coated fiber is slightly higher than the acrylate-coated fiber, the much lower Weibull slope indicates that the defect distribution is much broader, resulting in a greater range in failure strengths. Also shown in Figure 5 are the data for the same fiber structure $(275 / 302 \mu \mathrm{m})$ that was supplied by Polymicro without a loose outer jacket.

\section{Fiber with 200- $\mu m$ Core Diameter}

Tests were also made on a PolymicroTechnologies fiber, FVA 200/240/480 $\mu m$, which had an acrylate buffer. Twenty samples were tensile-tested to failure using a free-fiber length of 0.5 meter. The Weibull plot is provided in Figure 6, and it can be seen that the average strength at failure, $614 \mathrm{Kpsi}$, is close to the strength for the 100/125/250 $\mu \mathrm{m}$ fiber (see Figure 3) with acrylate buffer. 


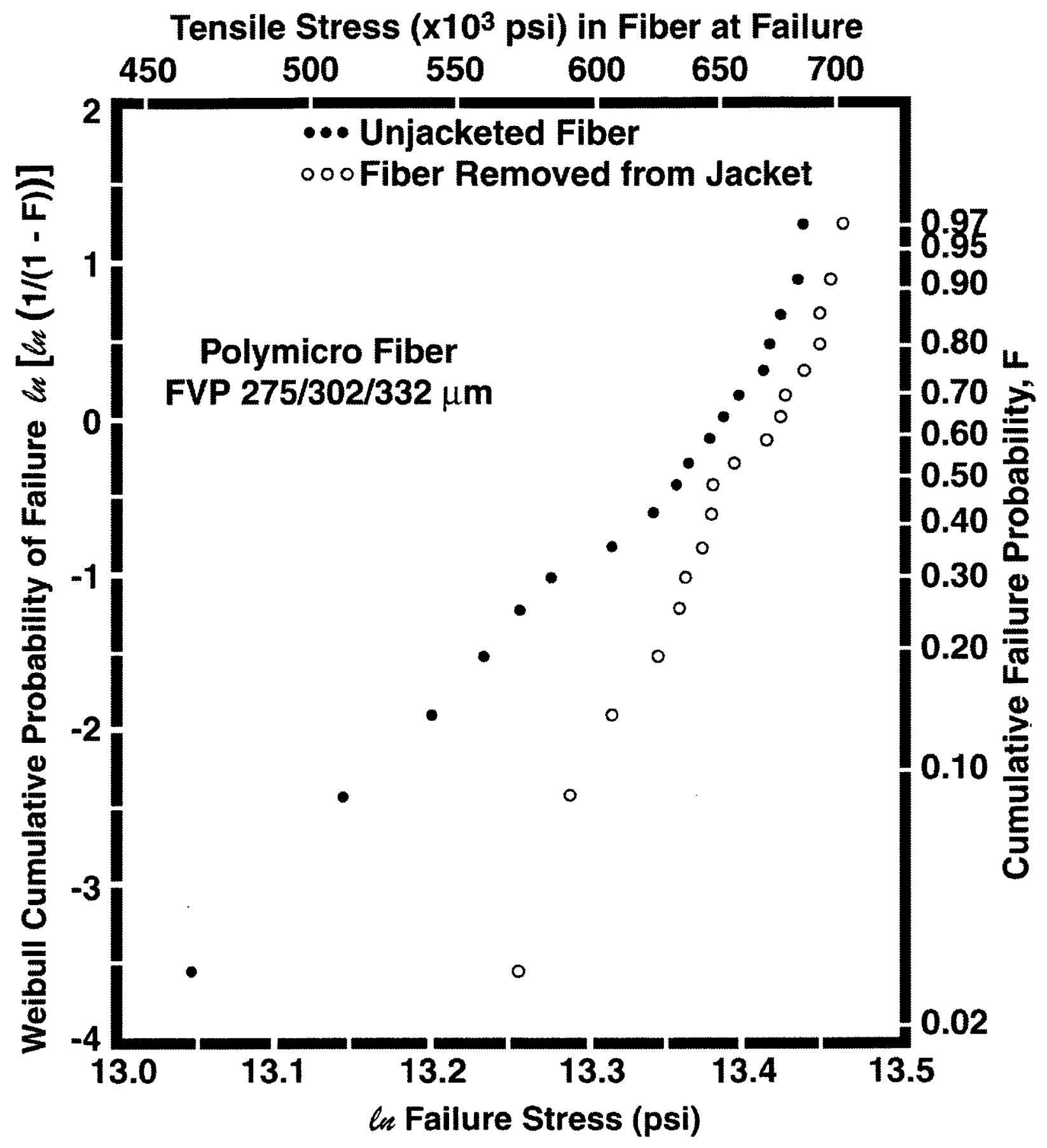

Figure 5. Weibull Cumulative Failure Probability Versus Logarithm of Fracture Stress for 275- $\mu m$ Core Fiber With Polyimide Buffer 
ln Fracture Stress (psi)

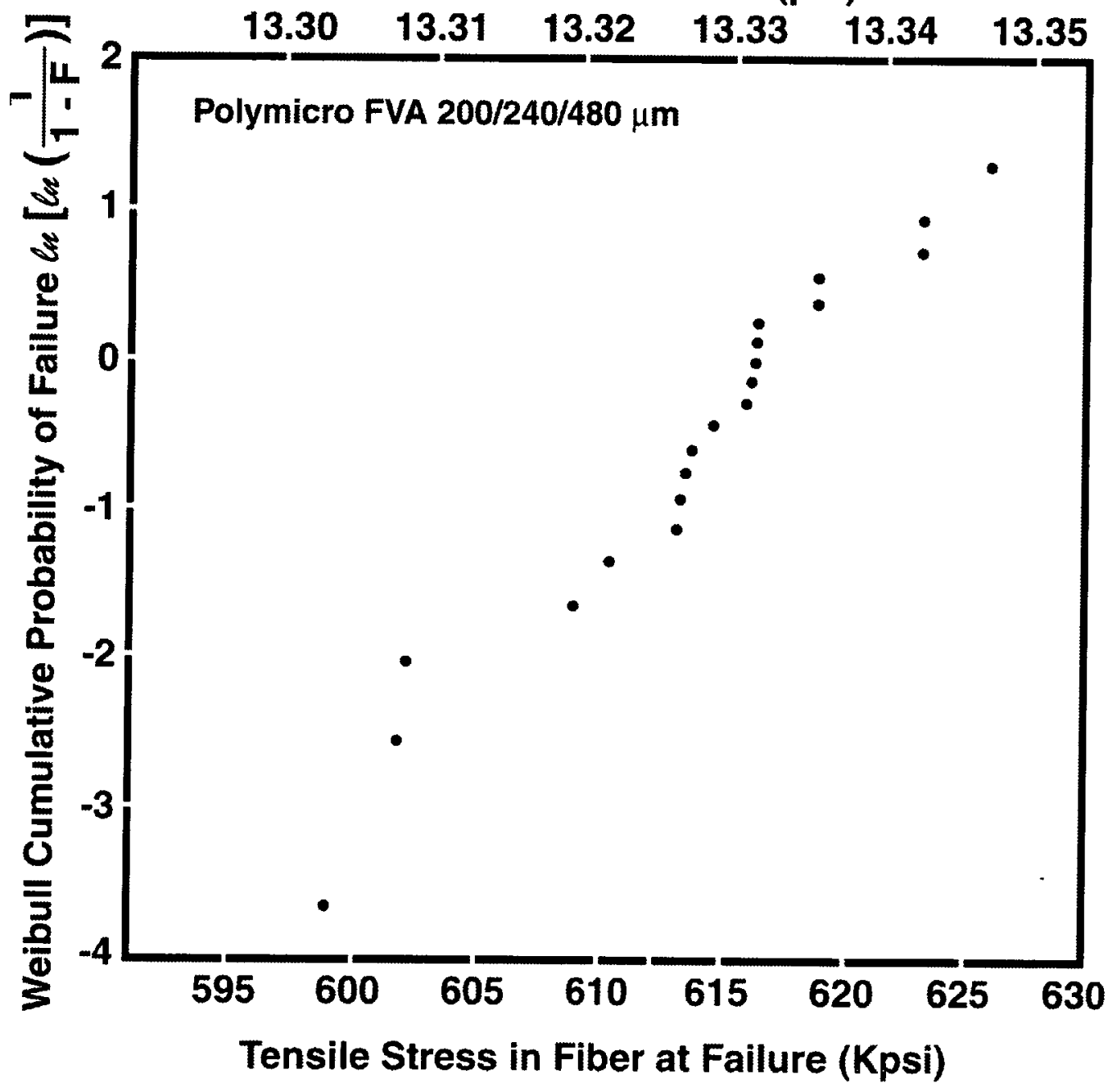

Figure 6. Weibull Cumulative Failure Probability Versus Logarithm of Fracture Stress for 200- $\mu \mathrm{m}$ Core Fiber With Acrylate Buffer 


\section{Fibers with 350- $\mu m$ and 365- $\mu m$ Core Diameter}

The buffer material serves not only to protect the fiber from inadvertent abrasion or handling damage, but also it acts as a barrier in preventing moisture from reaching the silica surface. When surface flaws or minute cracks are exposed to moisture, a corrosion action takes place that weakens the bonds and results in crack growth and, ultimately, failure of the glass fiber. The presence of moisture at defect sites is particularly harmful when the region of the fiber is placed under applied bending or tensile stress because the stress-corrosive action proceeds at an accelerated rate. Therefore, the success of a given buffer material in preventing moisture from arriving at the silica surface is of great importance to fiber lifetime under stress.

Fibers from two different companies, having different buffer materials, were tested for strength. This work was for the program, for which a fiber core of approximately $365 \mu \mathrm{m}$ was desired. SpecTran provided a fiber designated by $350 / 385 / 415 / 750$ $\mu \mathrm{m}$, indicating silica core and silica cladding of diameters $350 \mu \mathrm{m}$ and $385 \mu \mathrm{m}$, respectively. For a moisture barrier, SpecTran applied a carbon coating directly on the silica cladding surface, which in turn was coated with polyimide to an outer diameter of $415 \mu \mathrm{m}$. This in turn was coated with Hytrel (polyester elastomer) to an outer diameter of $750 \mu \mathrm{m}$. Very close in core diameter was a fiber marketed by 3M Specialty Optical Fibers, West Haven, CT, designated by FG-365-UER. The core diameter was $365 \mu \mathrm{m}$, and the cladding was $400 \mu \mathrm{m}$. The fiber contained a hard fluoropolymer (TECS) coating with outer diameter $430 \mu \mathrm{m}$, which in turn was coated with a Tefzel (fluorinated polymer) buffer of outer diameter $730 \mu \mathrm{m}$.

A total of 16 SpecTran fiber samples were tested to failure in tension using the Instron, and 10 fiber samples from $3 \mathrm{M}$ were tensile-tested to failure. Plots of the Weibull cumulative failure probability versus the natural logarithm of the fiber stress at failure are given in Figure 7 . The $3 \mathrm{M}$ fiber not only has a higher average strength at failure $(715 \mathrm{Kpsi})$ compared to the SpecTran fiber ( $340 \mathrm{Kpsi}$ ), but the variation in tensile strength values for $3 \mathrm{M}$ is much less than that of the SpecTran fiber. This is evidenced by the fact that the Weibull slope calculated from the data of Figure 7 for the $3 \mathrm{M}$ fiber $(m=139)$ is much larger than that for the SpecTran fiber $(m=26)$. Discussions with SpecTran regarding the low tensile strengths and accompanying variation revealed a possible source. According to SpecTran, the application of the carbon coating directly on the silica cladding surface is accomplished by a chemical vapor deposition process and this, they believe, can result in surface irregularities on the silica that initiate failure under applied stress. In any event, whatever advantages the carbon might provide as a moisture barrier, the SpecTran composite structure is characterized by low tensile strength accompanied by relatively large variation in strength, compared to the $3 \mathrm{M}$ fiber. 


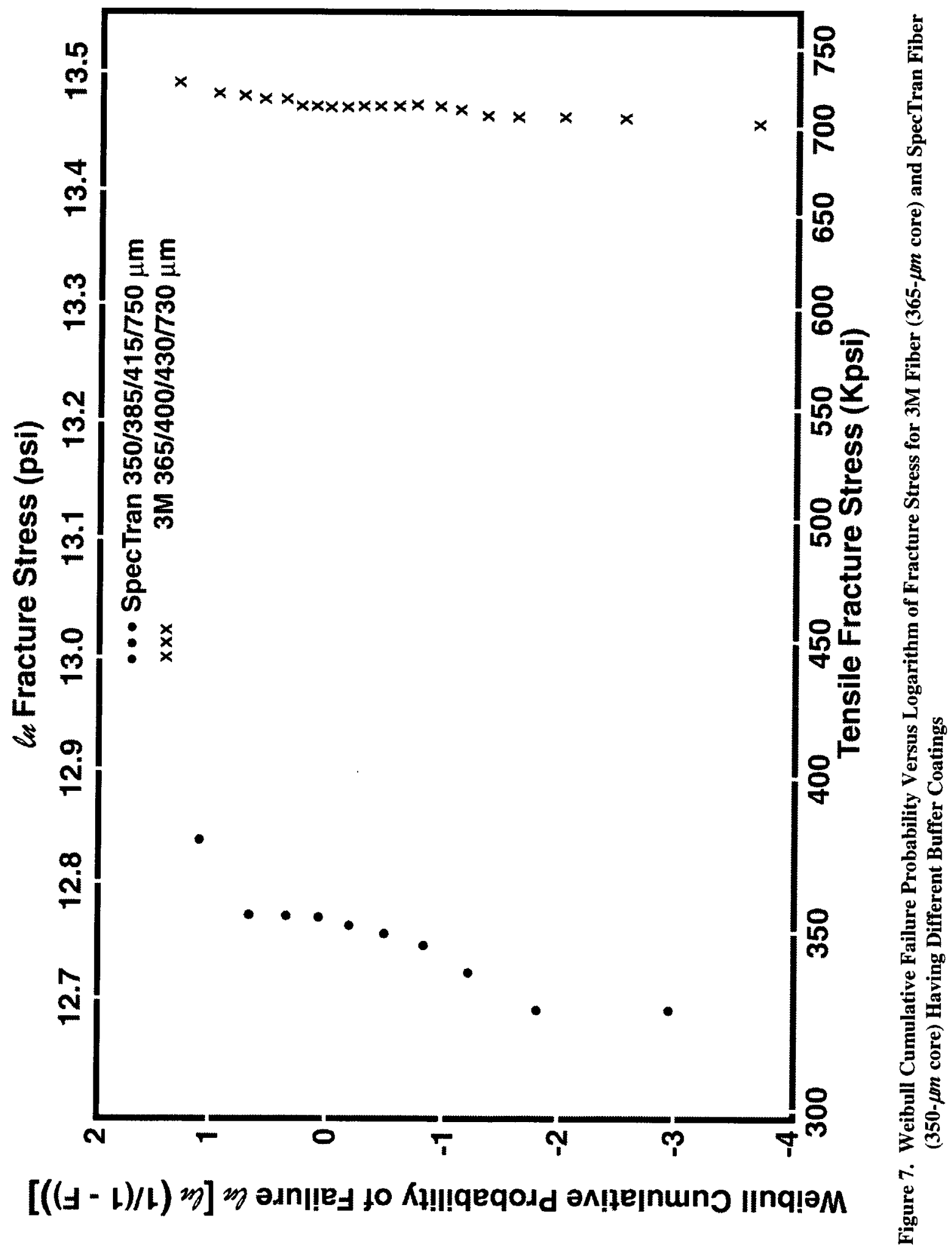


Tensile failure testing was also done with free-fiber gauge lengths of $2.5 \mathrm{~m}$ on both the $3 \mathrm{M}$ and SpecTran fibers. The Weibull cumulative failure probabilities versus fracture stress for the 2.5- $m$ lengths are given in Figure 8 for the $3 \mathrm{M}$ fiber, along with the results from Figure 7 for free test lengths of $0.5 \mathrm{~m}$. Results for the SpecTran fiber are given in Figure 9. For the 3M fiber, the mean fracture stresses and Weibull slopes are very close in value at $2.5-m$ lengths compared to 0.5- $m$ lengths. However, for the SpecTran fiber, the average tensile strength at failure is 12.5 percent lower for the 2.5- $m$ lengths than for the 0.5- $m$ lengths, and the Weibull slope is approximately 44 percent lower for $2.5 \mathrm{~m}$ versus $0.5 \mathrm{~m}$.

\section{Stress Aging}

\section{Bending Stress}

A test was performed to determine the effect of bend-stress aging on the subsequent tensile strength of the $3 \mathrm{M}$ optical fiber. Note from Figure 7 that the average tensile stress at failure is $714.5 \mathrm{Kpsi}$, with the minimum stress at failure being $680 \mathrm{Kpsi}$ for as-received $3 \mathrm{M}$ fiber. A value of bending stress to use during the stress aging process was chosen at approximately 70 percent of the average tensile stress, or approximately $495 \mathrm{Kpsi}$. A conveniently available rod diameter on which to wrap one-meter lengths of fiber to induce a peak bending sstress in the fiber cross section near the value $495 \mathrm{Kpsi}$ is 0.313 -inch diameter. The calculated peak bending stress in the $3 \mathrm{M}$ fiber wrapped tightly on a 0.313 -inch diameter rod is $493.8 \mathrm{Kpsi}$. Accordingly, one-meter lengths of each of 13 fibers were wrapped on 0.313-inch diameter rods. A couple of feet of unwrapped fiber was provided at each end so that the fibers could later be unwrapped from the rods and tested for tensile strength in the Instron in a configuration in which the wrapped onemeter length corresponded in full to the free-fiber test length. All 13 stressed fibers were maintained inside a Plexiglas enclosure in which a saturated solution of potassium acetate was used to maintain a relative humidity level of 23 percent. $^{8}$ The temperature was measured at 70 $72^{\circ} \mathrm{F}$ throughout the stress-aging process. The aging was continued uninterrupted for 170 hours, slightly longer than one week. After 170 hours of continuous stress aging, the fibers were unwrapped and mounted, sequentially, in the Instron for tensile testing to failure. A plot of the Weibull probability of failure versus failure stress for the 13 stress-aged fiber samples is given in Figure 10. Also included in Figure 10 are additional data of the type given in Figure 7 for the $3 \mathrm{M}$ fiber in the as-received condition, that is, without stress aging.

From the data of Figure 10, it can be seen that the stress aging has two effects. First, the tensile strength of the stress-aged fiber is lower and, secondly, the Weibull slope is substantially lower. A lower Weibull slope indicates that the defect distribution, leading to failure in tension, is wider than in the non-aged fiber. The data of Figure 10 represent an aging process associated essentially with stress alone, without moisture, because the relative humidity was maintained relatively low at 23 percent with the aid of the potassium acetate. ${ }^{8}$ 


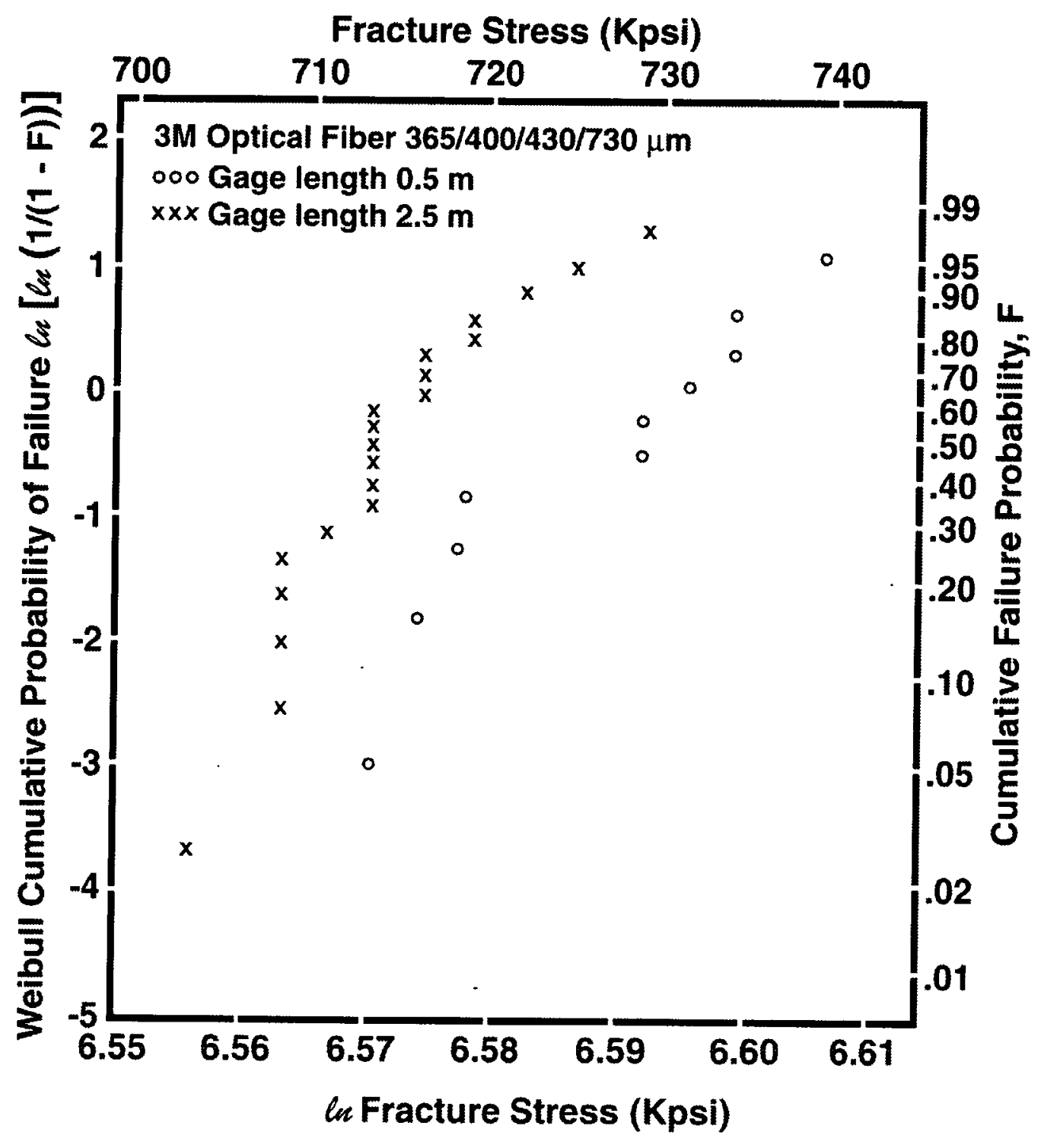

Figure 8. Weibull Cumulative Failure Probability Versus Logarithm of Fracture Stress at Two Gauge Lengths for $3 \mathrm{M}$ Fiber 


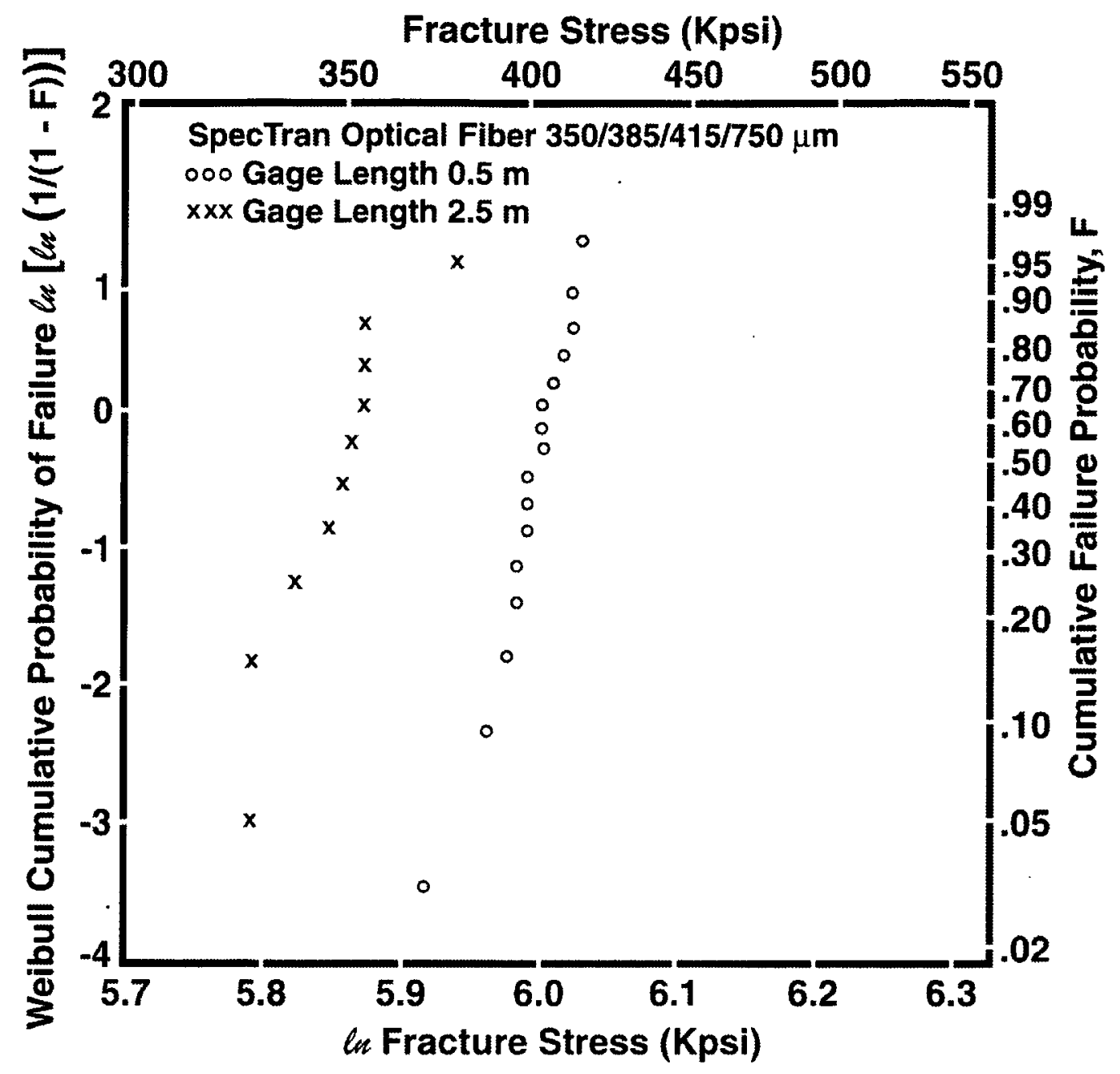

Figure 9. Weibull Cumulative Failure Probability Versus Logarithm of Fracture Stress at Two Gauge Lengths for SpecTran Fiber 


\section{M Optical Fiber: FG-365-UER}

$x x x$ Fiber As-Received

... Fiber Aged at $493.8 \mathrm{Kpsi}$ Bending Stress for 170 hrs., $23 \%$ Relative Humidity, $7^{\circ}{ }^{\circ} \mathrm{F}$

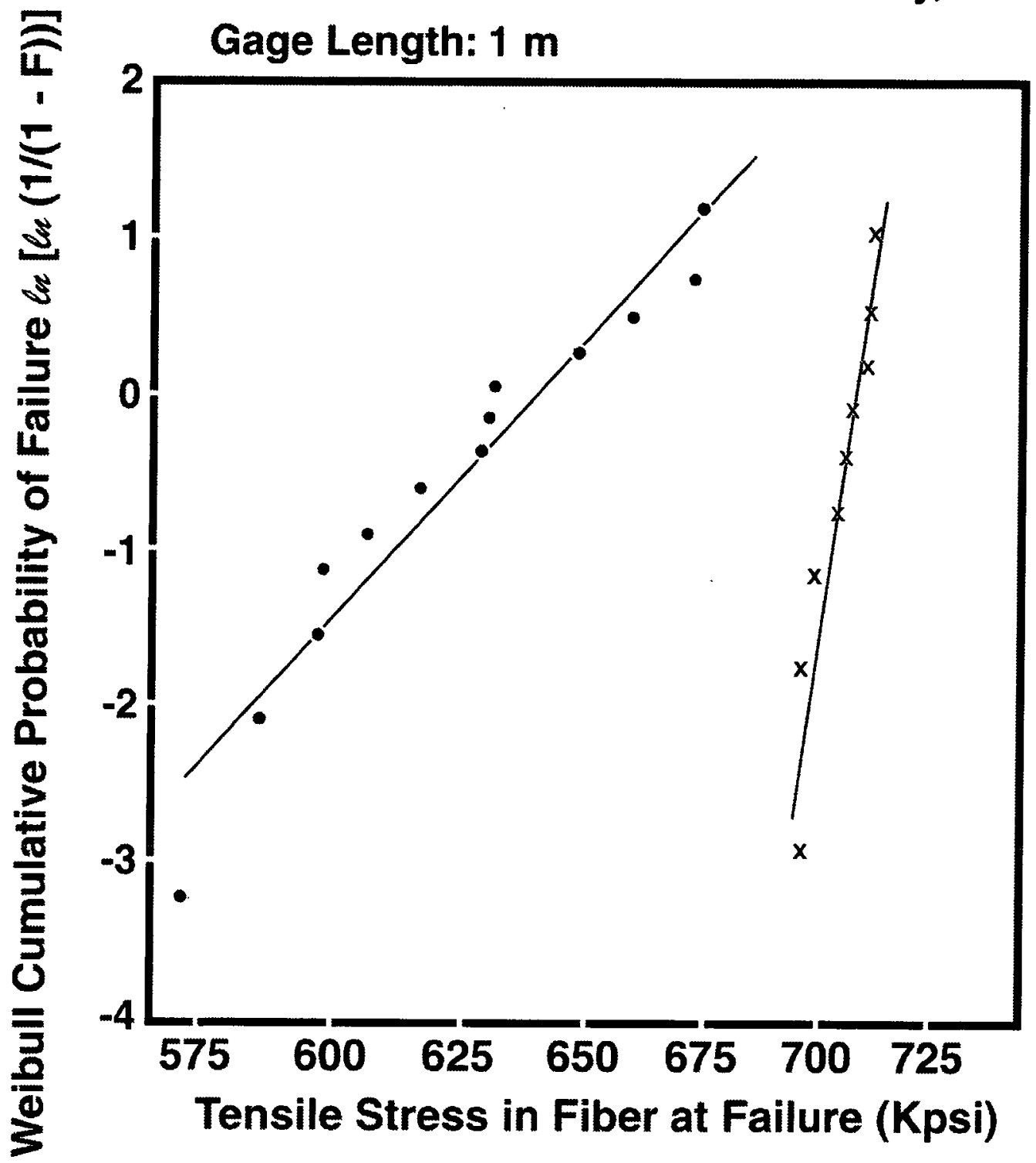

Figure 10. Weibull Cumulative Failure Probability Versus Logarithm of Fracture Stress for 3M Fiber After Aging at 493.8 Kpsi Bend Stress for 170 Hours at Relative Humidity of 23 Percent, $\mathbf{7 0 - 7 2}^{\circ} \mathrm{F}$ 


\section{Tensile Proof at $250 \mathrm{Kpsi}$}

Another type of stress aging involves tensile stress and can be done during the process of tensiletesting to failure. Proof-testing done by fiber manufacturers involves passing the fiber over pulleys of appropriately chosen diameter so that the induced bending stress in the fiber is of the magnitude desired as "proof." During this process, the linear speed of the fiber passing around the pulley is relatively high so that any given segment of the fiber is subjected to bending stress for only a brief time interval, normally less than one second. ${ }^{7}$ Therefore, any surface defects that are present do not have time to grow under the locally applied bending stress, whereas any defect that cannot withstand the proof bending stress will simply cause fracture of the fiber.

Segments of optical fiber can also be subjected to a tensile proof test using the Instron. To do this, a segment of fiber mounted in the Instron is subjected to loading at a prescribed rate, up to a load corresponding to the desired tensile proof stress, and then the load is quickly removed. In this way, the fiber is subjected to the maximum, proof tensile stress for only the brief time just as the maximum is reached. However, this method of proofing differs from the continuous passage of fiber over pulleys used by fiber manufacturers in that the entire free length of the fiber in the Instron is subjected to loading during the time interval over which the stress is increased from zero to the maximum value. This means that all defects present on the fiber surface are subjected to applied stress over the cumulative time of loading.

The results of a particular type of stress aging testing on the 3M fiber are presented in Figure 11. First, a number of fibers were tensile-tested to failure in the standard way, that is, beginning in the as-received condition; and the solid dots in Figure 11 represent the Weibull failure probability. Next, each of a group of fibers was proof-tested to a tensile stress of $250 \mathrm{Kpsi}$ in the Instron, with the load being released immediately upon reaching the stress level of $250 \mathrm{Kpsi}$. Each fiber was then tensile-tested to failure in the standard way, and the Weibull failure probabilities are represented by the open circles in Figure 11. Note that the average failure stress of these samples is slightly, but significantly, lower than that of the samples tested in the asreceived state (solid dots).

\section{Tensile Stress Dwell at $250 \mathrm{Kpsi}$}

Each of several fiber samples was loaded to a tensile stress level of $250 \mathrm{Kpsi}$ and allowed to remain at this level for an aging interval of 10 seconds. After this, the loading was reduced to zero and, then, the sample was tensile tested to failure in the standard way. The data for the latter are represented by the crosses in Figure 11. Note that the stress aging for 10 seconds at $250 \mathrm{Kpsi}$ results in a further degradation in average stress at failure. Compared to the asreceived state, both the tensile proof test at $250 \mathrm{Kpsi}$ and the 10 -second aging at $250 \mathrm{Kpsi}$ allow sufficient time for intrinsic defects to grow, thereby resulting in lower average failure strength in subsequent testing. 


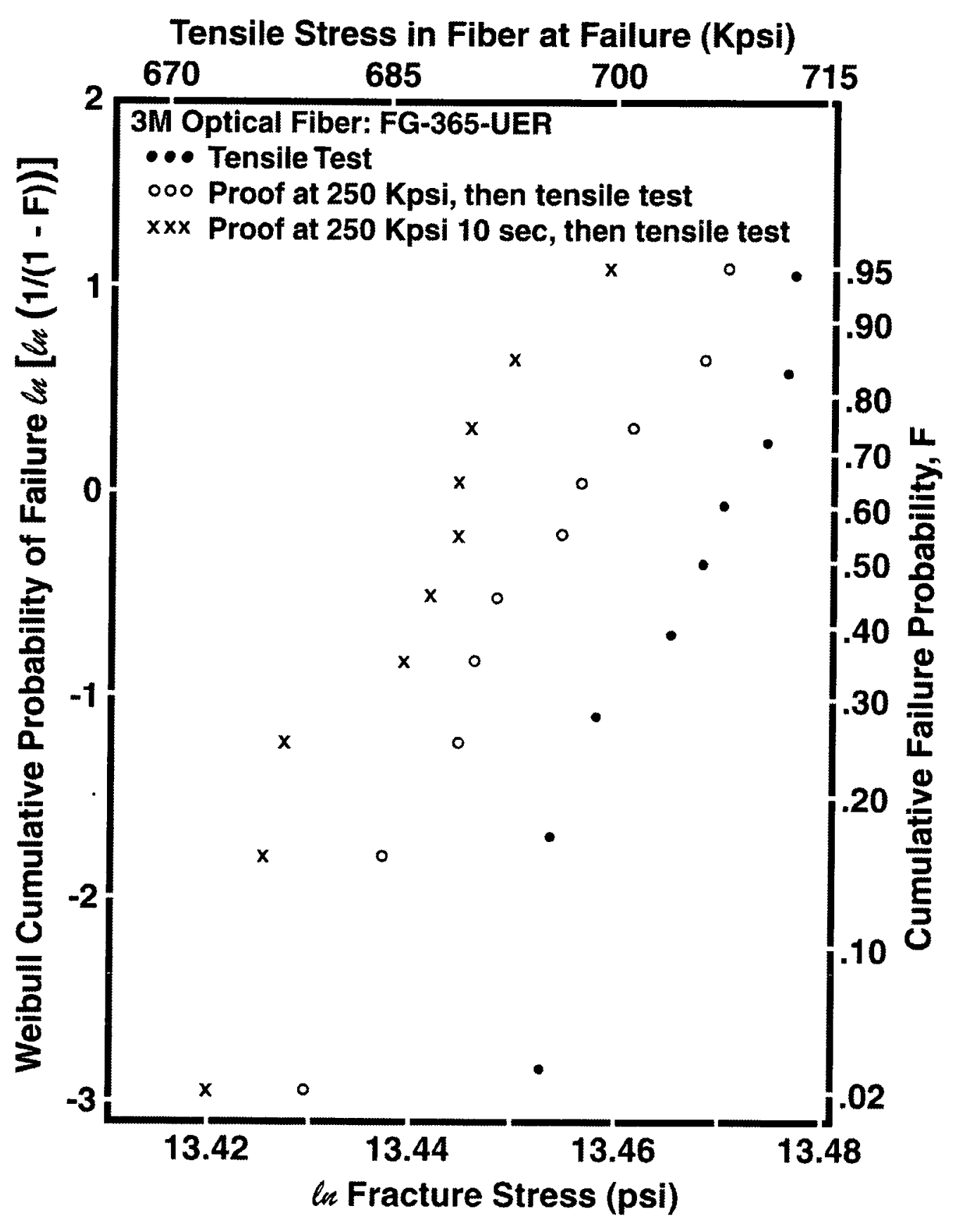

Figure 11. Effects of Proof-Test Aging on Weibull Cumulative Failure Probability, Versus Logarithm of Fracture Stress, for 3M Fiber 


\section{Tensile Strain Rate}

A further indication that defects grow in time under applied stress is provided by tensile testing to failure at the standard strain rate of 4 percent of the length per minute and comparing the results with the same fiber tested at a much higher strain rate. This work was done with 1-meter test lengths on Polymicro FVA 200/240/480- $\mu m$ fiber. The Weibull plot of the data obtained at the standard rate of 4 percent of length per minute $(1.6 \mathrm{in} . / \mathrm{min})$ on the $1-m$ samples is given by the dots in Figure 12. Results of tests on the same fiber done with a strain rate of 10.6 percent of length per minute ( 4.24 inches/min) are shown by the crosses in Figure 12. The Weibull slopes of the two data sets are essentially the same, but the average strength at failure is significantly higher $(640 \mathrm{Kpsi})$ for the fiber tested at the high strain rate compared to that $(614 \mathrm{Kpsi})$ of the fiber tested at the standard, lower strain rate. In fact, as can be seen from the data in Figure 12, the lowest stress at failure in the high strain rate testing is higher than the highest stress at failure in the group tested at the standard, but lower rate of strain. At the higher rate of strain, the cumulative dwell time to reach a given proof stress level is smaller than at lower applied strain rates, and hence defects do not have as long to "grow" under stress.

\section{Tensile Testing of Laminated Fiber}

Some potential applications may require a number of fibers side-by-side in a kind of ribbon structure. One way to form a ribbon is through the use of Kapton adhesive tape and a laminating process. An immediate question is that regarding strength of the fiber after laminating. To get a preliminary answer, the Polymicro fiber $275 / 302 / 332 \mu \mathrm{m}$ was chosen. This fiber has a polyimide buffer that will withstand $400^{\circ} \mathrm{C}$, much higher than acrylate buffer which will decompose at $150^{\circ} \mathrm{C}$. The polyimide buffer is necessary because laminating temperatures are approximately $365^{\circ} \mathrm{F}$ with Kapton adhesive tape. Accordingly, ten fibers were laminated over a length of $0.5 \mathrm{~m}$, allowing 2 feet unlaminated fiber length at either end for subsequent mounting in the Instron for tensile testing. A schematic of a laminated fiber is shown in Figure 13. For lamination, the fiber is sandwiched between two pieces of adhesive Kapton tape, each of which is 0.0015-inch thick prior to lamination. The $275 / 302 / 332-\mu \mathrm{m}$ fiber has an outer polyimide buffer diameter of 0.0131 inch. After laminating for 1 hour at $365^{\circ} \mathrm{F}$ and approximately $25 \mathrm{psi}$, the measured thickness was $0.0148 \mathrm{inch}$, indicating that some of the adhesive thickness had re-distributed. These laminated samples were tensile-tested to failure. The mean failure stress was found to be 670.9 $K p s i$, and the Weibull slope was calculated as 15.6. This mean failure stress is slightly higher than the data of Figure 5 for this fiber, and the Weibull slope is intermediate to the slopes for the jacketed and unjacketed data for 275/302/332- $\mu \mathrm{m}$ fiber in Figure 5. It would appear that the laminating process did not degrade the Polymicro 275/302/332- $\mu \mathrm{m}$ polyimide-coated fiber. 


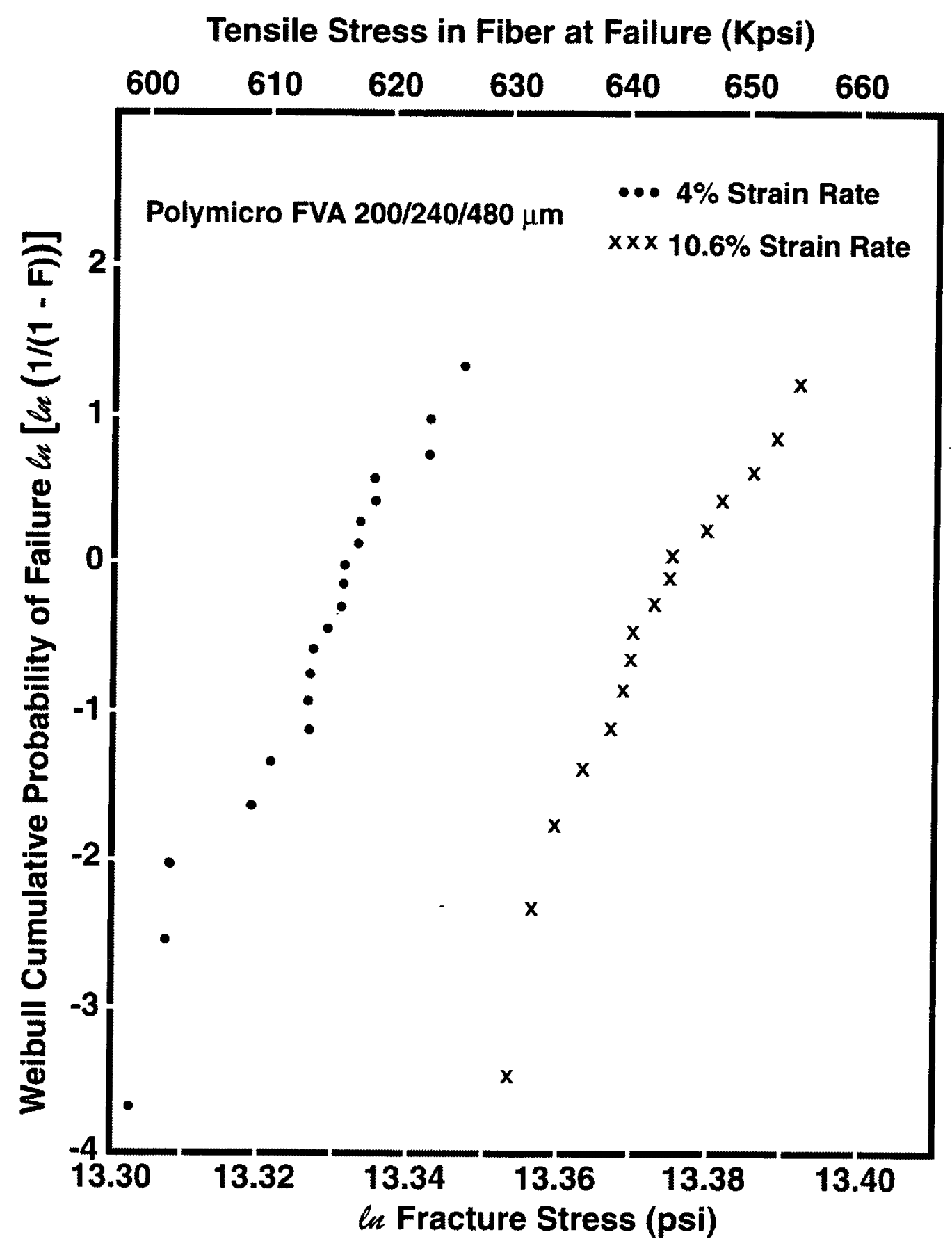

Figure 12. Effect of Tensile Strain Rate on Weibull Cumulative Failure Probability, Versus Logarithm of Fracture Stress, on 200- $\mu m$ Core Fiber 


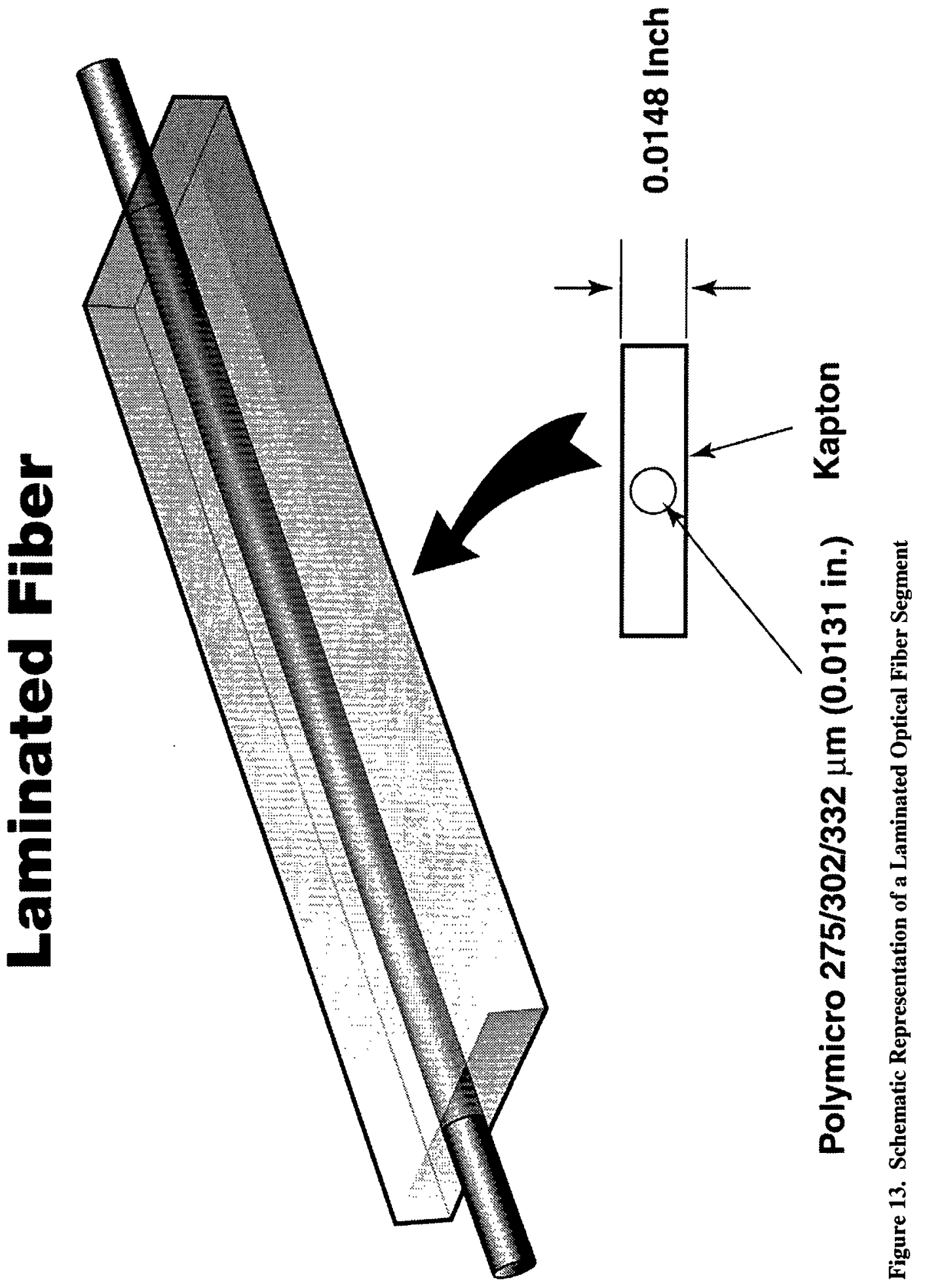




\section{Elastic Modulus}

During tensile testing of fibers with the Instron, a graph of fiber elongation, $\Delta l$, versus applied tensile load, $F$, is provided. From this, the elastic modulus $E$ can be calculated from the relation $E=(F / A) /\left(\Delta l / l_{o}\right)$, where $A$ is the glass fiber cross section and $l_{o}$ is the initial fiber length. If $F$ is taken as the load at failure and $\Delta l$ the corresponding change in fiber length, then the calculated value of $E$ represents an average over-the-load interval at failure. Using the measured elongation at the failure load of 140 pounds, the calculated elastic modulus over the interval for the silica fiber is $E=10.15 \times 10^{6}$ psi.

In practice, the value of $E$ for glass is taken as $10.2 \times 10^{6} \mathrm{psi} .{ }^{9}$ Some workers have reported that the elastic modulus of silica increases slightly with increasing levels of strain present in the silica. In the present work, the Instron data of fiber elongation versus applied tensile load can be used to calculate the fiber elastic modulus as a function of strain, $\Delta l / l_{o}$, in the fiber. To do this, a representative plot of applied tensile load and resulting fiber elongation obtained during the tensile testing of 2.5- $\mathrm{m}$ samples of the $3 \mathrm{M}$ fiber was used. As can be seen from earlier data in Figure 7, the average tensile failure of the $3 \mathrm{M}$ fiber occurred at $714.5 \mathrm{Kpsi}$, and this corresponded to a maximum applied load $F \cong 140 \mathrm{lbs}$. Accordingly, the overall load interval was divided into seven intervals, each of 20 pounds. From the Instron data of fiber displacement versus load, the actual loads at the beginning and end of each interval were known, as well as the change in elongation, or change in strain, over the interval and, therefore, the elastic modulus could be calculated as a function of strain. A best-fit straight line to the data gives the result $E=E_{o}(1+a \in)$, where $E_{o}$ is the zero-strain value of elastic modulus, $\epsilon$ is the strain $\Delta l / l_{o}$, and $a$ has the value $a=1.49$. Others have reported a value of $a=3$ for silica fiber. ${ }^{9}$ One reason the estimate of $a=1.49$ is lower than the value reported by others may be that the measured fiber elongation in the present case includes a component of elongation in the fiber segments wrapped about both capstans in the Instron. That is, no extra precaution was taken to prevent this from occurring. If it did occur to some extent, then the elongation registered by the Instron was actually greater than that of the free length being tested. This would cause the calculated strain $\epsilon \equiv \Delta l / l_{o}$ to be slightly high, resulting in a smaller value of $a$. In any event, this factor did not in any way influence the measured load at fiber failure and hence the calculated failure stress. 


\section{Fiber Time-to-Failure Under Applied Bending Stress}

Following the discussion connected with and leading to Equation 12, a series of tests was planned to measure the time-to-failure of fiber at various known bending stresses corresponding to reasonably short times, from which a meaningful extrapolation could be made to lower bending stress related to fiber survivability of 20 years or more. The plan was to choose smooth steel rods of various diameters and wrap one-meter lengths of the $3 \mathrm{M}$ fiber on the rods and then measure the times-to-failure at each of the rod diameters. The arrangement is represented schematically in Figure 14. To obtain statistically meaningful data, 10 fibers were wrapped at each of the diameters. The maximum bending stress in the fiber cross section wrapped on a rod of radius $R$ was calculated from the relation $\sigma=\epsilon \cdot E$ where $\epsilon$ is the strain in the bent fiber given by $\epsilon=r /(R+c+r)$, in which $r$ is the silica cladding radius $(200 \mu \mathrm{m})$ and $c$ is the coating wall thickness $(730 \mu m-400 \mu m) / 2$ for the $3 \mathrm{M}$ fiber. The strain, and therefore the stress, varies with the rod diameter and, consequently, the elastic modulus $E$ used to calculate the stress in the fiber from the relation $\sigma=\epsilon \cdot E$ must be determined from the relation $E=E_{o}(1+3 \in)$ for the level of strain corresponding to a given rod diameter. To provide relative short times-to-failure, smalldiameter rods were chosen to create high-bending stresses. As the study progressed, the results of the measured times-to-failure at given diameters were then used to estimate the rod diameter that would correspond to failure times of approximately one year. In all, six different rod diameters were used corresponding to six different induced bending stress levels in the fibers. The rod diameters were $0.281,0.312,0.330,0.343,0.375$, and 0.413 inch. For all except the largest rod diameter, the induced bending stresses were such that timers were used to measure the time-to-failure in each of the ten fibers wrapped at each of the five rod diameters. This was done by continuously passing light through each fiber and using a timer to record fiber failure as evidenced by decreased photo-detector response when fiber breakage occurred. For the smallest bending stress employed, with the 0.413 -inch diameter rod, failure times were sufficiently long that timing the failure to within one day of elapsed time gave an accuracy of 0.3 percent, without timers.

Upon completion of all the data, the average time-to-failure of the ten fibers at each rod diameter was calculated, along with the bending stress for the given rod diameter. Following the discussion related to Equation 12, a plot of the natural logarithm of time-to-failure, $\ln t_{f}$, versus the natural logarithm of the applied bending stress is given in Figure 15. In all, 60 fibers were involved in the study, 10 at each of the 6 stress levels. The placement of the short horizontal bars above and below the central average time indicate the high and low measured times at the given stress level. 


\section{Sleeve on Motor Shaft}

Plexiglas Block

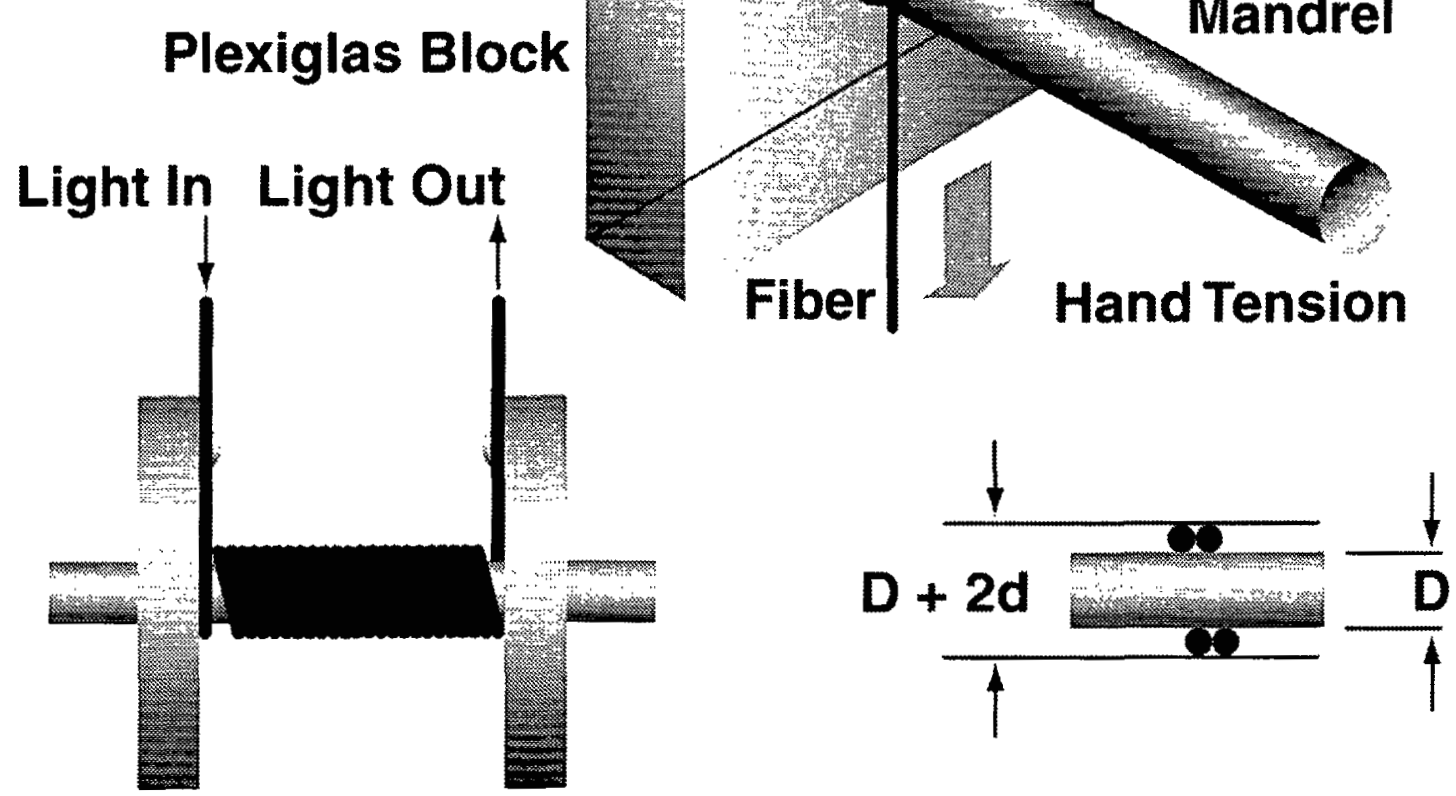

Mandrel

Figure 14. Method for Inducing Bending Stress in Fiber and Monitoring Time to Failure

The equation of the best-fit straight line through the data points in Figure 15 is

$$
\ln t_{f}=-23.851 \ln \sigma+324.917 \sec
$$

where $\sigma$ is bending stress in psi and $t_{f}$ is failure time in seconds. (Note that $n$ has the value 23.851.) The linear fit to the data is very good, with a correlation coefficient of 0.9992 . The relationship in Equation 13 can be used to calculate the bending stress corresponding to a timeto-failure of 20 years, for example. If Equation 13 is solved for $\sigma$ with a time of $t_{f}=20 y r s\left(6.3072 \times 10^{8} \mathrm{sec}\right)$ substituted, the result is 


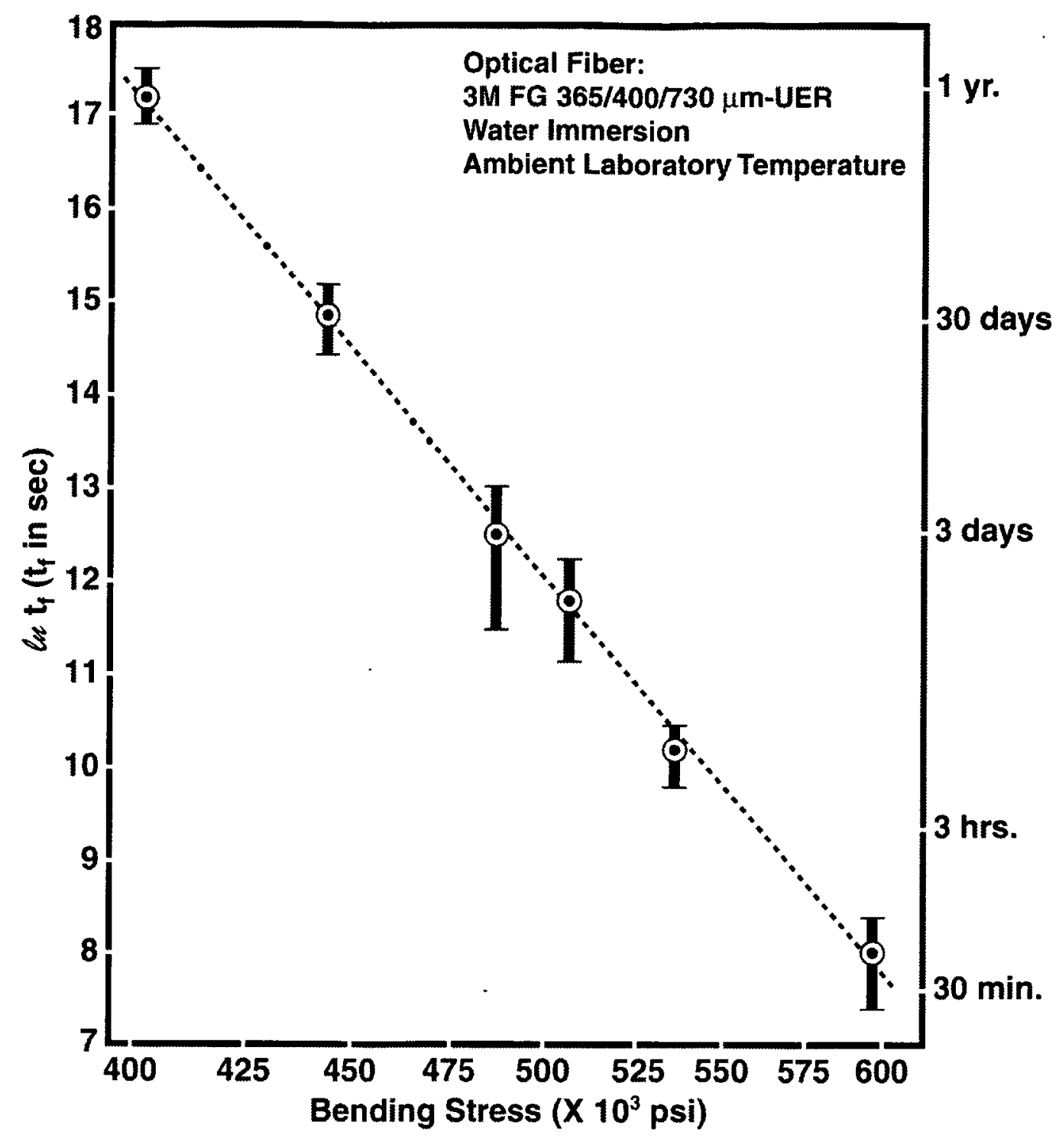

Figure 15. Plot of Logarithm of Mean Time-to Failure Versus Logarithm of Applied Bending Stress for 3M Fiber, 365- $\mu m$ Core Diameter 


$$
\sigma_{20}=352.654 \mathrm{Kpsi} \text {. }
$$

To determine the bend radius corresponding to the stress in Equation 14, the corresponding strain in the fiber must be known. The strain can be calculated by noting that $\sigma_{20}=E \cdot \epsilon_{20}$, but $E=E_{o}\left(1+a \epsilon_{20}\right)$, and hence

$$
\sigma_{20}=E_{o}\left(1+a \epsilon_{20}\right) \epsilon_{20}
$$

which leads to the quadratic in strain

$$
\epsilon_{20}^{2}+\frac{\epsilon_{20}}{a}-\frac{\sigma_{20}}{a E_{o}}=0
$$

Of the two roots to this quadratic, the one that applies is

$$
\epsilon_{20}=-1 / 2 a+\left(1 / a^{2}+4 \sigma_{20} / a E_{o}\right)^{1 / 2} / 2 \text {. }
$$

Using the value $E_{o}=10.2 \times 10^{6} \mathrm{psi}$ for zero-strain elastic modulus and the value $a=3$ in Equation 16, along with the calculated bend stress $\sigma_{20}$ from Equation 14, gives the value of strain

$$
\epsilon_{20}=0.0316 \text {. }
$$

Therefore, from the relation $\epsilon=r /(R+c+r)$, the bend radius $R_{20}$ that corresponds to the stress level $\sigma_{20}$ in Equation 14, and for which the fiber is expected to remain intact for $t_{f}=20 y r s$, can be determined as

$$
R_{20}=\frac{r}{\epsilon_{20}}-c-r
$$

Substituting $r=200 \mu m$ and $c=(730 \mu m-400 \mu m) / 2$ for the $3 \mathrm{M}$ fiber gives

$$
R_{20}=5.968 \mathrm{~mm} \text {. }
$$


The latter minimum bend radius is based on Equation 13, which is a best fit to all the data in Figure 15, spanning failure times as short as 30 minutes and as great as 1 year. Self-consistency of the data can be demonstrated by using only those data points in Figure 15 representing failure times from 30 minutes to 30 days to calculate a bend radius for which fiber survivability of 20 years would be expected. Accordingly, the best-fit linear relationship representing the data of Figure 15 over the failure times from 30 minutes to 30 days is

$$
\ln t_{f}=-23.493 \ln \sigma+320.184 \text {, }
$$

with a correlation of 0.9985 .

Setting $t_{f}=20 y r s$ and calculating the corresponding bending stress gives $\sigma_{20}=350.258 \mathrm{Kpsi}$. Solving for the corresponding strain and then the bend radius yields

$$
R_{20}=6.007 \mathrm{~mm}
$$

which is very close (within 0.65 percent) to that determined from all the data from 30 minutes to 1 year, which resulted in $R_{20}$ in Equation 19.

\section{$\underline{\text { Tabulation of Results }}$}

A comparative summary of all the analyses associated with the tensile testing of the various fibers and the tensile aging studies is provided in Table 1. 
Table 1. Compilation of Results Obtained from Tensile Testing

\begin{tabular}{|c|c|c|c|c|c|c|}
\hline \multicolumn{3}{|c|}{ Optical Fiber Description } & \multicolumn{3}{|c|}{$\begin{array}{l}\text { Analysis of Tensile } \\
\text { Test Results }\end{array}$} & \multirow[t]{6}{*}{$\begin{array}{c}\text { Distinguishing Factors Related to } \\
\text { Fiber History, Construction, or } \\
\text { Pre-Conditioning }\end{array}$} \\
\hline Mfg. & $\begin{array}{l}\text { Core/Cladding } \\
\text { Diameter }(\mu m)\end{array}$ & Buffer & $\begin{array}{l}\text { Gauge } \\
\text { Length* }\end{array}$ & $\begin{array}{l}\text { Mean } \\
\text { Stress at } \\
\text { Failure }\end{array}$ & $\begin{array}{l}\text { Weibull } \\
\text { Slope }\end{array}$ & \\
\hline Polymicro & $100 / 125$ & Acrylate & $1 m$ & $604.9 \mathrm{Kpsi}$ & 44.5 & \\
\hline SpecTran & $100 / 125$ & Tefzel & $1 \mathrm{~m}$ & 680.9 Kpsi & 47.5 & \\
\hline Polymicro & $100 / 125$ & Acrylate & $2.5 \mathrm{~m}$ & $608.7 \mathrm{Kpsi}$ & 122.6 & \\
\hline SpecTran & $100 / 125$ & Tefzel & $2.5 \mathrm{~m}$ & $679.5 \mathrm{Kpsi}$ & 214.9 & \\
\hline Polymicro & $275 / 302$ & Polyimide & $1 m$ & 653 Kpsi & 20.7 & Removed from jacket. \\
\hline Polymicro & $275 / 302$ & Polyimide & $1 \mathrm{~m}$ & 614 Kpsi & 11.1 & Supplied by Polymicro, unjacketed. \\
\hline Polymicro & $200 / 240$ & Acrylate & $0.5 \mathrm{~m}$ & 614 Kpsi & 105.5 & \\
\hline $\begin{array}{l}\text { 3M } \\
\text { (FG-365-UER) }\end{array}$ & $365 / 400$ & Tefzel & $2.5 \mathrm{~m}$ & $714.5 \mathrm{Kpsi}$ & 139 & High $-\mathrm{OH}$. \\
\hline $\begin{array}{l}\text { SpecTran } \\
\text { (SF Hi-OH 350/ }\end{array}$ & $\begin{array}{c}350 / 385 \\
/ 385 / 415 / 750)\end{array}$ & Polyimide & $2.5 \mathrm{~m}$ & $348.3 \mathrm{Kpsi}$ & 25.8 & $\begin{array}{l}\text { CVD carbon coating on cladding } \\
\text { followed by Polyimide at } 415 \mu \mathrm{m} \text {, } \\
\text { then Hytrel (polyester elastomer) to } \\
750 \mu \mathrm{m} \text {. }\end{array}$ \\
\hline $\begin{array}{l}3 \mathrm{M} \\
\text { (FG365-UER) }\end{array}$ & $365 / 400$ & Tefzel & $\begin{array}{l}0.5 m \\
2.5 m\end{array}$ & $\begin{array}{l}726.4 \mathrm{Kpsi} \\
714.6 \mathrm{Kpsi}\end{array}$ & $\begin{array}{l}111 \\
136\end{array}$ & \\
\hline $\begin{array}{l}\text { SpecTran } \\
\text { (SF Hi-OH 350/ }\end{array}$ & $\begin{array}{c}350 / 385 \\
1 / 385 / 415 / 750) \\
\end{array}$ & Polyimide & $\begin{array}{l}0.5 \mathrm{~m} \\
2.5 \mathrm{~m}\end{array}$ & $\begin{array}{l}400 \text { Kpsi } \\
348 \text { Kpsi }\end{array}$ & $\begin{array}{l}44.1 \\
24.9\end{array}$ & \\
\hline $\begin{array}{l}3 \mathrm{M} \\
\text { (FG 365-UER) }\end{array}$ & $365 / 400$ & Tefzel & $\begin{array}{l}1 \mathrm{~m} \\
1 \mathrm{~m}\end{array}$ & $\begin{array}{l}705 \mathrm{Kpsi} \\
625 \mathrm{Kpsi}\end{array}$ & $\begin{array}{l}122.6 \\
22.5\end{array}$ & $\begin{array}{l}\text { As-received from } 3 \mathrm{M} \text {. } \\
\text { Stress-aged } 170 \mathrm{hrs} \text { at } 493.8 \mathrm{Kpsi} \text {, } \\
23 \% \text { rel. hum., } 72^{\circ} \mathrm{F} \text {. }\end{array}$ \\
\hline $\begin{array}{l}3 \mathrm{M} \\
\text { (FG 365-UER) }\end{array}$ & $365 / 400$ & Tefzel & $\begin{array}{l}1 m \\
1 m \\
1 m\end{array}$ & $\begin{array}{l}705 \mathrm{Kpsi} \\
694.9 \mathrm{Kpsi} \\
686.6 \mathrm{Kpsi}\end{array}$ & $\begin{array}{l}122.6 \\
91.7 \\
97.9\end{array}$ & $\begin{array}{l}\text { As-received from 3M. } \\
\text { Proof at } 250 \mathrm{Kpsi} \text {, reduce to } 0 \text {, then } \\
\text { test. } \\
\text { Proof at } 250 \mathrm{Kpsi} \text {, dwell } 10 \mathrm{sec} \text {, } \\
\text { reduce to } 0 \text {, then test. }\end{array}$ \\
\hline Polymicro & $200 / 240$ & Acrylate & $\begin{array}{l}1 m \\
1 m\end{array}$ & $\begin{array}{l}614 \text { Kpsi } \\
642 \text { Kpsi }\end{array}$ & $\begin{array}{l}105.5 \\
107.9\end{array}$ & $\begin{array}{l}4 \% \text { of length/min strain rate. } \\
10.6 \% \text { of length/min strain rate. }\end{array}$ \\
\hline Polymicro & $275 / 302$ & Polyimide & $0.5 \mathrm{~m}$ & $670.9 \mathrm{Kpsi}$ & 15.6 & $\begin{array}{l}\text { Laminated between two pieces } \\
\text { Kapton tape } 365^{\circ} \mathrm{F}, 1 \mathrm{hr}, 25 \mathrm{psi} \text {. }\end{array}$ \\
\hline
\end{tabular}

\footnotetext{
*Unless otherwise noted, the strain rate during testing is 4 percent of gauge length per minute.
} 
Based on a 20-year fiber lifetime, Table 2 provides a summary of the extrapolated value of radius to which the $3 \mathrm{M}$ fiber can be bent and survive. These results are based on the data embodied in Figure 15 and the associated calculations related to Equations 13 through 21.

Table 2. Extrapolated Minimum Fiber Bend Radius for 20-Year Lifetime (3M FG 365-UER)

\begin{tabular}{|c|c|}
\hline Static Bend Stress Data Base & Extrapolated Radius \\
\hline 30 minutes -1 year & $5.968 \mathrm{~mm}$ \\
30 minutes - 30 days & $6.007 \mathrm{~mm}$ \\
\hline
\end{tabular}

\section{Accomplishments}

The mechanical strength of optical fiber obtained from different sources was investigated with the aid of tensile testing. The tensile fracture data was analyzed with the aid of Weibull failure probability. The Weibull analysis provides not only the average strength at failure, but also the dispersion in strength, as determined by the slope of the Weibull plot of cumulative failure probability versus failure stress. The analysis showed that two fibers can have very nearly the same average strength, but fiber with an acrylate protective buffer coating shows less variability in strength than fiber with a polyimide (Kapton-like) buffer coating. Also, the study showed that carbon, applied to silica as a moisture buffer coating, not only results in wider dispersion in fiber strength, but also reduces very significantly the average tensile strength.

Fiber segments containing polyimide buffer coating were laminated with Kapton adhesive laminate. The fibers in the 0.5 -meter long laminate were tensile-tested to failure. The laminating process did not degrade the average tensile strength, compared to unlaminated fiber of the same type, and the dispersion in tensile strength values was not affected.

Various stress aging studies were done. It was found that subjecting $3 \mathrm{M}$ fiber (365- $\mu m$ core/400$\mu m$ cladding) to a bending stress of $493.8 \mathrm{Kpsi}$ for 170 hours, with the fiber maintained at 23 percent relative humidity at $72^{\circ} \mathrm{F}$, caused a significant decrease in average fiber strength and increased variability in strength values upon subsequent tensile testing. In other studies, samples of the $3 \mathrm{M}$ fiber were subjected to a proof load at $250 \mathrm{Kpsi}$, with no dwell at this level. Other fiber samples from $3 \mathrm{M}$ were subjected to $250 \mathrm{Kpsi}$ for 10 seconds after which the load was reduced to zero. In subsequent tensile testing to failure, both sets of stress-aged fibers showed reduced average strength and greater variability in strength values. 
Knowledge of the fiber elongation versus applied tensile load during tensile testing provided a means of calculating the elastic modulus of the glass fiber. Calculated values over the range of 140 pounds for the $3 \mathrm{M}$ fiber gave $E=10.15 \times 10^{6} \mathrm{psi}$, which is in agreement with reported values. Moreover, the data showed that the calculated elastic modulus increases slightly with the level of strain present in the fiber cross section; and an increase of this type has been reported by others.

A database was developed relating the time-to-failure of the $3 \mathrm{M}$ fiber subjected to bending stresses at six different levels. The failure times ranged from approximately 30 minutes to approximately 1 year among the various bend-stress levels. Based on a best-fit analytic relationship representing the data, with a correlation of 99.92 percent, an extrapolation was made to lower bending stresses and corresponding longer time-to-failure of the fiber subjected to these lower bending stresses. The results showed, for example, that a failure time of 20 years corresponded to a bending stress associated with a minimum fiber bend radius of $5.968 \mathrm{~mm}$.

\section{Future Work}

Work is in progress to develop a database of time-to-failure for $100-\mu \mathrm{m}$ core optical fiber subjected to bending stress of different levels. From this data, extrapolations will be made to determine minimum bending radius of this fiber for survival of 20 years. 


\section{References}

1. T. A. Michalske and S. W. Freiman, "A Molecular Interpretation of Stress Corrosion in Silica," Nature, Vol. 295, No. 5849, 1982, pp 511-512.

2. W. Weibull, “A Statistical Distribution Function of Wide Applicability," Journal of Applied Mechanics, Vol. 18, No. 9, 1951, pp 293-297.

3. F. Bacon and M. Maklad, "Long Term Mechanical Reliability of Single Mode Optical Fibers," SPIE, Vol. 2292 Fiber Optic and Laser Sensors XII (1994), pp 340-352.

4. B. Bergman, "On the Estimation of the Weibull Modulus," Journal of Materials Science Letter, No. 3, (1984), pp 689-692.

5. G. Evans, "Analysis of Strength Degradation After Sustained Loading," Journal of the American Ceramic Society, Vol. 57, No. 9, 1974, pp 410-411.

6. R. J. Charles, "Static Fatigue of Glass: I, II," Journal of Applied Physics, Vol. 29, No. 11, pp 1657-1662, (1958).

7. FOTP-28. Fiber Optic Test Procedure for Measuring Dynamic Tensile Strength of Optical Fibers, EIA/TIA, 2001 Pennsylvania Ave., Washington, D.C. 20006.

8. Standard Practice for Maintaining Constant Relative Humidity by Means of Aqueous Solutions, American Society for Testing and Materials, ASTM E104-85.

9. S. T. Gulati, J. D. Helfinstine, G. S. Glaesemann, R. D. Roberts, E. Cuellar, and L. M. Middleman, "Improvements in Optical Fiber Reliability via High Fatigue Resistant Composition," SPIE 842 Fiber Optics Reliability: Benign and Adverse Environments, pp 2230 (1988). 
